\title{
REVISIÓN Y ACTUALIZACIÓN DEL MÉTODO SIMPLIFICADO DE ANÁLISIS DE ESTRUCTURAS DE MAMPOSTERÍA DE LOS REGLAMENTOS DE DISEÑO SÍSMICO DE MÉXICO
}

\author{
Arturo Tena Colunga ${ }^{(1)}$, Jesús Cano Licona ${ }^{(2)}$, Arturo López Blancas ${ }^{(3)}$ y Miguel Ángel Pérez Osornio ${ }^{(4)}$
}

\section{RESUMEN}

El método simplificado, muy utilizado en análisis y diseño sísmico de estructuras de mampostería, se basa en la distribución de fuerzas laterales en estructuras simétricas con diafragmas rígidos. En este artículo se presentan los aspectos más relevantes de las investigaciones realizadas para revisar a fondo las disposiciones del método simplificado y que han permitirlo actualizarlo, proporcionando con base en extensos estudios paramétricos, factores de área efectiva actualizados para tres distintos niveles de desempeño estructural, así como los respectivos valores límite de la excentricidad estática en planta $\left(e_{s}\right)$ para su aplicación. Estas propuestas ya se incorporan en el método simplificado del capítulo de diseño por sismo del nuevo Manual de Obras Civiles de Comisión Federal de Electricidad (MOC-2008).

Palabras clave: mampostería confinada, excentricidad estática límite, torsión, desempeño estructural, método simplificado de análisis sísmico.

\begin{abstract}
The simplified method for the seismic analysis (SMSA) and design for low-rise masonry structures with rigid diaphragms allowed by Mexican Building Codes is frequently used in the design practice of Mexico. In this paper, the most relevant aspect of a comprehensive research study devoted to do an in-depth review of the SMSA is presented. This research has allowed updating the SMSA. Based on extensive parametric studies, effective shear area factors $\left(F_{A E}\right)$ originally proposed in the SMSA are modified to improve the estimates of shear forces using this method. New $F_{A E}$ are proposed for three different performance levels for the structure, as well as their corresponding limit for a computed static torsional eccentricity in plan $\left(e_{s}\right)$. This updated version of the SMSA is already included in the seismic recommendation of the new Manual of Civil Structures (MOC-2008), a model seismic code of Mexico.
\end{abstract}

Artículo recibido el 1 de mayo de 2009 y aprobado para su publicación el 18 de enero de 2010. Se aceptarán comentarios y/o discusiones hasta cinco meses después de su publicación.

${ }^{1}$ Profesor, Departamento de Materiales, Universidad Autónoma Metropolitana Azcapotzalco, Av. San Pablo 180, Col. Reynosa

Tamaulipas, 02200 México, DF, e-mail: atc@,correo.azc.uam.mx

${ }^{2}$ Proyectista, Alonso y Asociados Proyecto Estructural, Carretera Federal México Toluca 1725-C5, Col. Lomas de Palo Alto, CP 05110 México, DF, e-mail: jesuscl@alonsoasociados.com.mx

${ }^{3}$ Proyectista, Gerencia Técnica, INPRESA, Calzada Desierto de los Leones 4073, 01060 México, D.F, e-mail:

ahur_inpresa@hotmail.com

${ }^{4}$ Proyectista, Grupo GEO, México, DF, e-mail: osornio31@yahoo.com 
Keywords: confined masonry, static eccentricity, torsion, structural performance, simplified method of seismic analysis.

\section{INTRODUCCIÓN}

El método simplificado de análisis y diseño fue originalmente propuesto en 1977 en las Normas Técnicas Complementarias de Estructuras de Mampostería (NTCM-77 1977) y para Diseño por Sismo (NTCS-77 1977) del Reglamento para Construcciones del Distrito Federal (RCDF-76). Desde ese entonces, el método simplificado ha sido la base para el análisis y diseño de estructuras de mampostería, ya que permite realizar su análisis y diseño de forma rápida y aproximada.

De acuerdo con las Normas Técnicas para Diseño por Sismo (NTCS) desde 1977, al aplicar el método simplificado de análisis se hará caso omiso de los desplazamientos horizontales, torsiones y momentos de volteo, y se verificará únicamente que en cada piso la suma de las resistencias al corte de los muros de carga, en la dirección que se considera la aceleración, sea cuando menos igual a la fuerza cortante que obre en dicho piso.

De lo establecido en las NTCS se puede concluir que el método simplificado de análisis se basa en la distribución de fuerzas laterales en estructuras con diafragmas rígidos (por ejemplo, Tena 2007) y donde la distribución de las rigideces laterales de sus elementos resistentes es totalmente simétrica y la carga lateral se aplica en una sola dirección, como se ilustra esquemáticamente en la figura 1. Bajo esta hipótesis, existen dos aspectos que se desprecian de manera importante y que son los efectos de torsión y los efectos bidireccionales. De hecho, el método simplificado de análisis desprecia la flexibilidad del diafragma, los efectos de torsión, los efectos bidireccionales, los momentos de volteo y los desplazamientos horizontales.

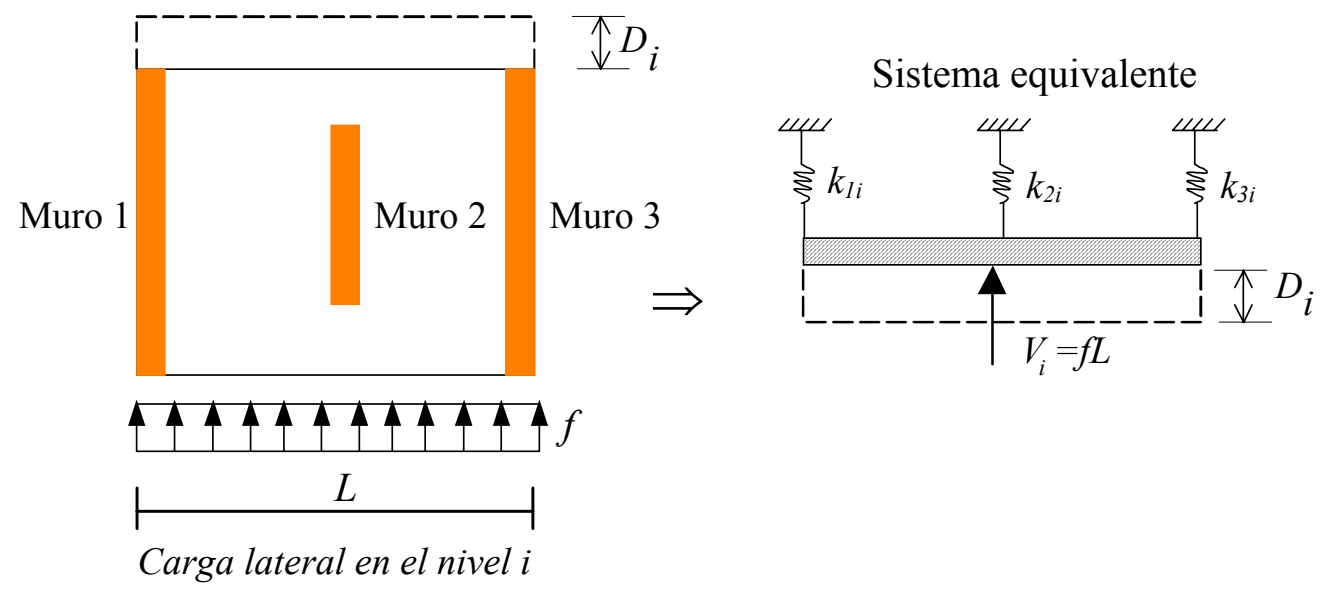

Figura 1. Distribución de fuerzas laterales en planta según el método simplificado

Con respecto a la posibilidad de flexibilidad del diafragma, la restricción en la relación de aspecto de la planta de la estructura parece acotar razonablemente esta condición con respecto a lo que se ha observado en algunos estudios paramétricos disponibles en la literatura para sistemas de piso de concreto reforzado con base en losas macizas o perimetralmente apoyadas. Recientemente, esta condición se revisó también para estructuras de mampostería que cumplen con el método simplificado y donde se usan 
sistemas de piso de vigueta y bovedilla, así como losas planas aligeradas con bloques de espuma de poliestireno (Cortés 2009, Tena y Cortés 2009).

En cuanto a los momentos de volteo, la restricción en la relación de esbeltez de la estructura también parece acotar razonablemente esta condición conforme a lo que se ha observado en algunos estudios paramétricos disponibles en la literatura (por ejemplo, Taranath 1988, Bazán y Meli 1998). Con respecto a los efectos bidireccionales, éstos pueden tomarse indirectamente con los coeficientes sísmicos reducidos que se establecen en las tablas respectivas de las NTCS y del Manual de Obras Civiles para estructuras del grupo B.

$\mathrm{Al}$ emplearse el método simplificado principalmente en estructuras rígidas con base en muros, el obviar el cálculo de desplazamientos horizontales parece razonable, aunque no estaría de más proponer un procedimiento aproximado para su cálculo en función de las rigideces de entrepiso aproximadas que se pueden calcular a partir del mismo método simplificado y compararlas con límites de distorsión congruentes con el sistema estructural y material que se utilice.

Sin embargo, de todos los efectos que se desprecian, era la torsión la que no se acotaba de manera ingenieril en las versiones de las NTCS y NTCM de 1977 a 1995, ya que según el texto de dichas normas, bastaba con que la distribución de muros en planta fuera "sensiblemente simétrica" con respecto a dos ejes ortogonales. Resultaba inadecuado y un poco imprudente que las NTCS y las NTCM utilizaran un término tan subjetivo como la palabra "sensiblemente" para establecer una limitante en la aplicación del método simplificado de análisis. El primer autor propuso desde ese entonces que sería más razonable establecer un límite en función de un parámetro ingenieril que pudiera estimarse fácilmente, como la excentricidad estática calculada en planta $\left(e_{s}\right)$.

Uno de los cambios importantes en las NTCS-2004 (2004) y las NTCM-2004 (2004) es que ya se ha puesto una restricción para la respuesta torsional, de manera que actualmente $e_{s}$ se limita a un diez por ciento de la dimensión en planta del entrepiso medida paralelamente a dicha excentricidad. Este límite también lo establecen las Normas Técnicas Complementarias por Sismo (NTCS-2004) para considerar a una estructura como regular, de acuerdo con el requisito 10 de su Apartado 6. Además, en las NTCM2004 se propone un método aproximado para el cálculo de $e_{s}$.

La propuesta del valor límite de $e_{s}$ de las NTCS-2004 y NTCM-2004 tiene justificación en que el método simplificado debe permitirse exclusivamente en estructuras que cumplan cabalmente con las condiciones de regularidad del Apartado 6 las NTCS-2004. De hecho, no existían estudios específicos que permitieran definir cuál debía ser el valor límite de la excentricidad torsional calculada estáticamente $\left(e_{s}\right)$ para poder utilizar el método simplificado de análisis, comparando las fuerzas cortantes obtenidas mediante este método con respecto a soluciones analíticas rigurosas donde los efectos de torsión se tomen en cuenta en la determinación de fuerzas cortantes.

Aunque en términos generales se puede afirmar que el método simplificado está conceptualmente bien concebido desde sus inicios, también es un hecho que la propuesta original del método simplificado no había sido calibrada ni comparada con análisis más rigurosos, donde se consideraran las rotaciones de los muros en los entrepisos, las deformaciones por cortante, la respuesta tridimensional, el efecto del agrietamiento de los muros en elevación y la torsión del conjunto. Por ello, existía una imperiosa necesidad de revisar y en su caso, actualizar las disposiciones del método simplificado de cara al nuevo milenio. En las siguientes secciones se resumirán los aspectos más relevantes de una serie de investigaciones que fueron encauzadas a este propósito. 


\section{FUNDAMENTOS TEÓRICOS DEL MÉTODO SIMPLIFICADO}

El método simplificado se basa en la distribución de fuerzas laterales de estructuras simétricas con diafragmas rígidos cuando la carga lateral se aplica en una sola dirección, como se ilustra en la figura 1. Bajo estas hipótesis, en un entrepiso iésimo $(i)$ cualquiera, el cortante aplicado en el entrepiso $V_{i}$ produce un desplazamiento uniforme $D_{i}$ el cual es impuesto a los muros resistentes en la dirección de análisis, por lo cual cada muro $j$ del nivel iésimo toma una fuerza cortante $V_{j i}$ proporcionalmente a su rigidez lateral $k_{j i}$ :

Bajo esta hipótesis, el cortante aplicado en cada entrepiso se distribuye entre los distintos muros o elementos resistentes proporcionalmente a su rigidez.

$$
\begin{aligned}
& V_{i}=\sum_{j=1}^{n} V_{j i}=\sum_{j=1}^{n} k_{j i} D_{i} ; \rightarrow D_{i}=\frac{V_{i}}{\sum_{j=1}^{n} k_{j i}} \\
& V_{j i}=k_{j i} D_{i}=\frac{k_{j i}}{\sum_{j=1}^{n} k_{j i}} V_{i}
\end{aligned}
$$

La rigidez lateral de un muro depende principalmente de su deformación por cortante. Por lo tanto, si las deformaciones por flexión se toman de manera indirecta a partir de la rigidez a cortante en términos de un factor de área efectiva (el cual puede ser entendido por algunos como un factor de ajuste), entonces la rigidez lateral $k_{j i}$ del muro $j$ del nivel iésimo se puede aproximar como el producto de su módulo de rigidez a cortante $G$ por su área efectiva a cortante dividida entre la altura de entrepiso $H_{i}$ :

$$
k_{j i}=\frac{G F_{A E_{j i}} A_{T_{j i}}}{H_{i}}
$$

donde $F_{A E j i}$ es el factor de área efectiva del muro $j$ del nivel iésimo propuesto por el método simplificado y $A_{T j i}$ es el área de su sección transversal. Por lo tanto, de las ecuaciones 2 y 3 se obtiene que la fuerza cortante que debe tomar el muro $j$ del nivel iésimo es:

$$
V_{j i}=V_{i} \frac{F_{A E_{j i}} A_{T_{j i}}}{\sum_{j=1}^{n} F_{A E_{j i}} A_{T_{j i}}}
$$

El factor de área efectiva $F_{A E}$ propuesto para el método simplificado en los reglamentos de diseño sísmico de México (por ejemplo, MOC-93 1993, NTCS-2004 2004, NTCM-2004 2004) depende de la relación de aspecto del muro (alto entre largo, $H / L$ ) y está dado por las siguientes expresiones:

$$
F_{A E}=1 \quad \text { si } \frac{H}{L} \leq 1.33
$$




$$
F_{A E}=\left(1.33 \frac{L}{H}\right)^{2} \quad \text { si } \frac{H}{L}>1.33
$$

Dado que la rigidez lateral de los muros depende principalmente de su rigidez a cortante, la rigidez lateral de los muros en elevación se puede discretizar mediante un modelo de cortante (figura 2), por lo que se pueden despreciar las rotaciones que experimentan los muros en los entrepisos. En virtud de lo anterior, la matriz de rigidez lateral $\left[K_{D}\right]_{j}$ del muro $j$ es tridiagonal y, para el caso ilustrado en la figura 2, estaría dada por:

$$
\left[K_{D}\right]_{j}=\left[\begin{array}{ccc}
k_{j k}+k_{j l} & -k_{j l} & 0 \\
-k_{j l} & k_{j l}+k_{j m} & -k_{j m} \\
0 & -k_{j m} & k_{j m}
\end{array}\right]
$$

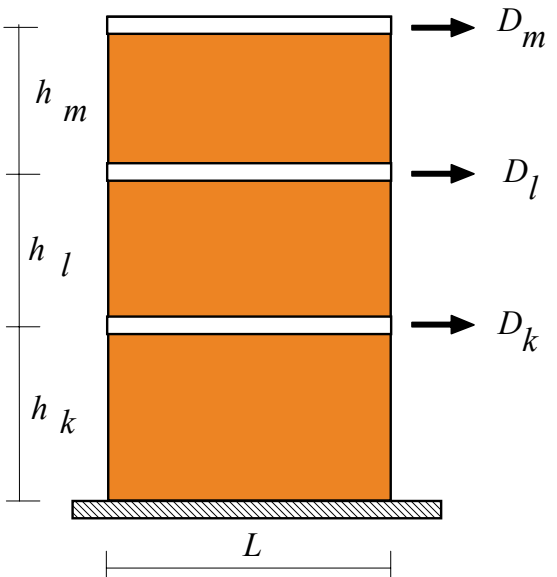

a) Muro de cortante

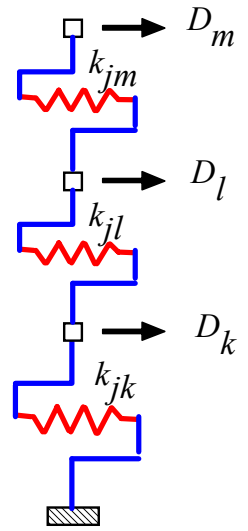

b) Modelo de cortante

Figura 2. Modelado de los muros en elevación bajo las hipótesis del método simplificado

Finalmente, la fórmula aproximada propuesta en el método simplificado de las NTCS-2004 y NTCM-2004 para estimar la excentricidad estática en planta para el nivel iésimo, $e_{s i}$, tiene sus bases en la determinación del centroide de las áreas efectivas de los muros con respecto al centro de cortante, como se ilustra en la figura 3, por lo que se calcula simplemente como:

$$
e_{s i}=\frac{\left|\sum_{j=1}^{n} x_{j} F_{A E j} A_{T j}\right|}{\sum_{j=1}^{n} F_{A E j} A_{T j}} \leq 0.1 B_{i}
$$




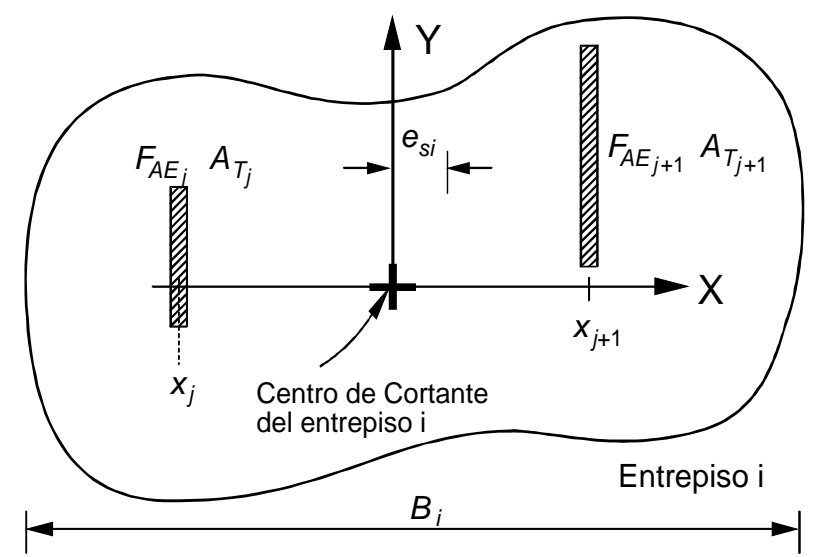

Figura 3. Requisito para evaluar la excentricidad estática en planta en el entrepiso $i, e_{s i}$ conforme al método simplificado (adaptado de NTCM-2004 2004).

\section{REVISIÓN INICIAL DEL MÉTODO SIMPLIFICADO}

Como ya se ha mencionado, el método simplificado está bien concebido conceptualmente desde sus inicios para el análisis y diseño de estructuras de mampostería de baja altura que sean simétricas y posean sistemas de piso rígidos y resistentes. Sin embargo, es un hecho que la propuesta original del método simplificado y sus factores de área efectiva no habían sido calibrados ni comparados con análisis más rigurosos, donde se consideraran las rotaciones de los muros en los entrepisos (figura 4), las deformaciones por cortante, la respuesta tridimensional o el efecto del agrietamiento de los muros en elevación.

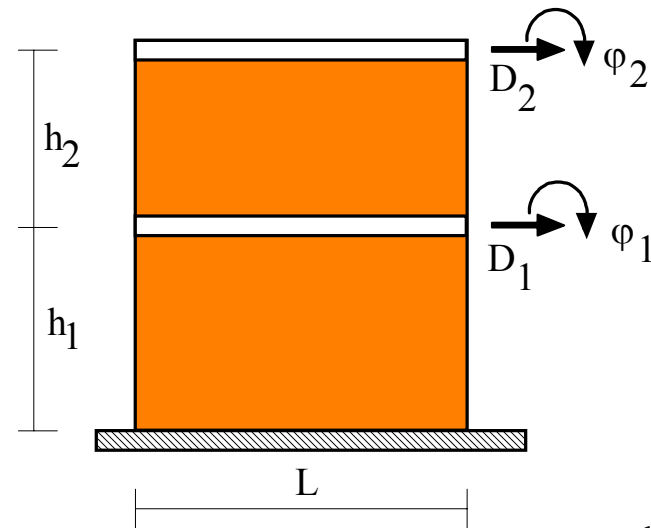

a) Muro sólido

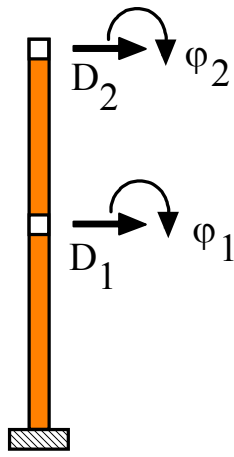

b) Columna ancha equivalente

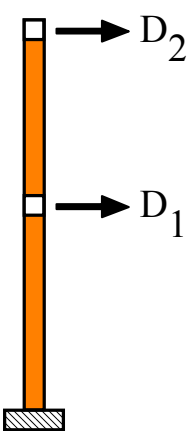

c) Viga condensada equivalente

Figura 4. Modelado de los muros en elevación considerando rotaciones y deformaciones por cortante

Por ello, se evaluó la importancia de las deformaciones por cortante de los muros en la ubicación de los centros de torsión de edificios mediante la determinación de excentricidades estáticas de cada entrepiso (Tena y Pérez 2000, Tena-Colunga y Pérez-Osornio 2005). Se compararon las diferencias que existen en las excentricidades estáticas si se consideran o no las deformaciones por corte, si se emplea la fórmula aproximada propuesta para el método simplificado por las NTCM-2004 y con las hipótesis sobre la matriz de rigidez lateral de muro conforme a las NTCM-2004. Se evaluó el caso común de edificios donde se tiene la misma distribución de muros en planta, pero en cada planta existen muros con distintas 
relaciones altura-longitud $(H / L)$, lo que ocasiona que el impacto de las deformaciones por cortante en la rigidez lateral de cada muro sea distinto (Figura 5).

Dicho estudio demuestra que las deformaciones por cortante tienen gran influencia en la ubicación de los centros de torsión de entrepiso de edificios que no sean totalmente simétricos en planta y en elevación. La ubicación de los centros de torsión puede variar notablemente entre los distintos entrepisos en edificios donde existan en planta muros con distintas relaciones $H / L$, a pesar de que dicha distribución de muros en planta sea idéntica en todos los niveles. Si dominan muros con relaciones de aspecto $H / L<1$ (muros cortos), las excentricidades estáticas calculadas tienden a incrementarse del último al primer nivel. Si dominan muros esbeltos $(H / L>1)$, las excentricidades estáticas calculadas tienden a incrementarse del primer al último nivel. Un estudio un poco más detallado del por qué sucede ésto ilustra que son precisamente el impacto que tienen las deformaciones por cortante en los grados de libertad de rotación de los muros lo que origina el cambio en los centros de torsión de entrepiso (Tena y Pérez 2000, TenaColunga y Pérez-Osornio 2005). De hecho, el impacto específico de las deformaciones por cortante en elevación para estructuras con la misma distribución de muros en planta es que ocasionan que, en lugar de tener un eje de rotación completamente vertical, como sucede en estructuras que se deforman esencialmente a flexión, se tenga un eje de rotación inclinado u oblicuo con respecto a la vertical.

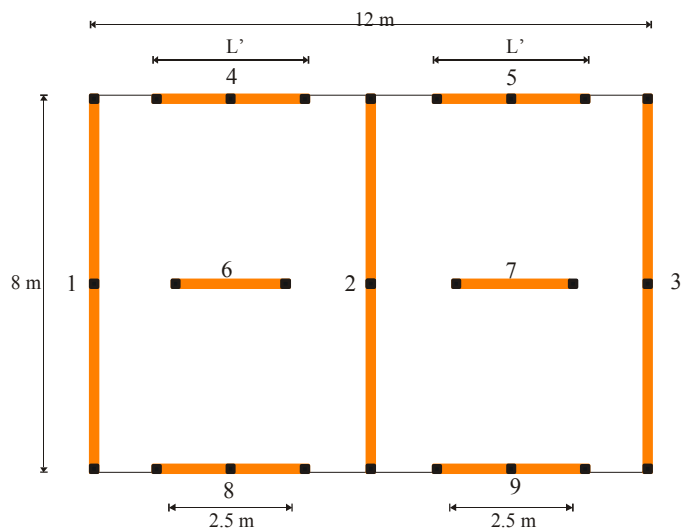

a) Planta tipo modelos simétricos

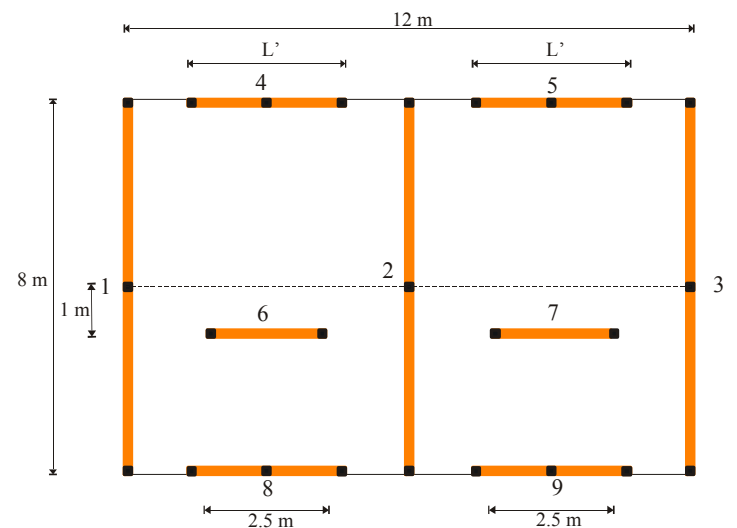

b) Planta tipo modelos asimétricos

Figura 5. Plantas tipo de los modelos estudiados por Tena y Pérez (2000)

El estudio también demuestra que la expresión aproximada propuesta por las NTCM-2004 para el cálculo de la excentricidad estática conforme al método simplificado da resultados razonables para modelos donde todos los muros tienen proporciones semejantes $(H / L)$. Para sistemas dominados por muros cortos $(H / L<1)$, la expresión es conservadora, mientras que para sistemas dominados por muros esbeltos $(H / L>1)$, la expresión no es conservadora.

También se estudió el impacto que en la distribución de las fuerzas cortantes atraídas por los muros tienen las deformaciones por cortante (Tena, Pérez y Cano 2002), por lo que se compararon las fuerzas cortantes que toman muros de mampostería con el método simplificado original con respecto a las obtenidas empleando un método riguroso de análisis tridimensional, tanto para sistemas simétricos como asimétricos (figura 5). En el estudio se consideró una relación de aspecto constante $H / L=1$ para los muros centrales (6 y 7), mientras que para los muros perimetrales $(4,5,8$ y 9 ) las relaciones de aspecto variaron, estudiándose las siguientes relaciones: $H / L=0.5,0.75,1,1.33$ y 2.0 , que son los valores identificados en las leyendas de la figura 5. El estudio demostró que pueden existir diferencias importantes entre las fuerzas cortantes de entrepiso estimadas con el método simplificado original cuando se comparan con respecto a métodos rigurosos de análisis estructural tridimensional cuando los muros tienen distintas 
relaciones de aspecto $(H / L)$, aun para sistemas simétricos (figura 6), por lo que se debían proponer factores de área efectiva $\left(F_{A E}\right)$ más realistas a como impactan las deformaciones por cortante en el análisis en todo el intervalo de relaciones de aspecto de los muros $(H / L)$, con base en estudios paramétricos. En los resultados mostrados en la figura 6a para sistemas simétricos se observa que cuando todos los muros (centrales y perimetrales) tienen la misma relación de aspecto $(H / L=1)$, entonces la aproximación del método simplificado es exacta con respecto a un análisis tridimensional riguroso, lo que no sucede cuando los muros en planta tienen diferentes relaciones de aspecto $(H / L)$, que sería el caso más común.

De estos resultados se concluyó que, para fines de análisis, sería deseable calibrar primero, de mejor manera, el factor $F_{A E}$ que proponen las NTCM-2004. Estos estudios se resumen en la siguiente sección.

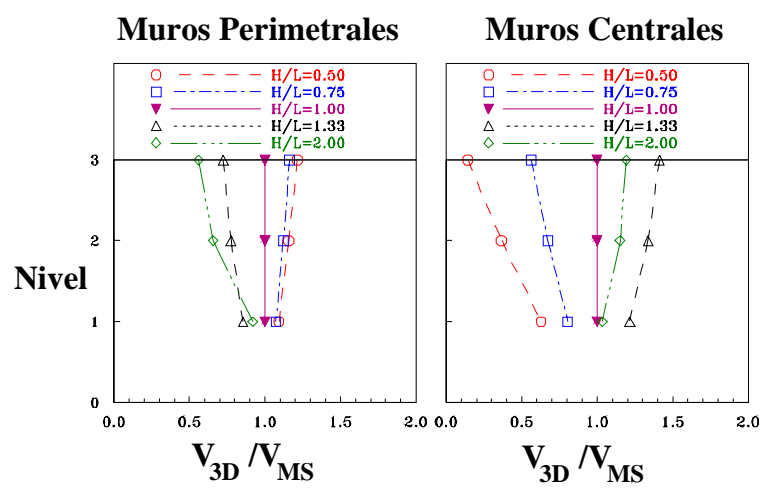

a) Modelos simétricos

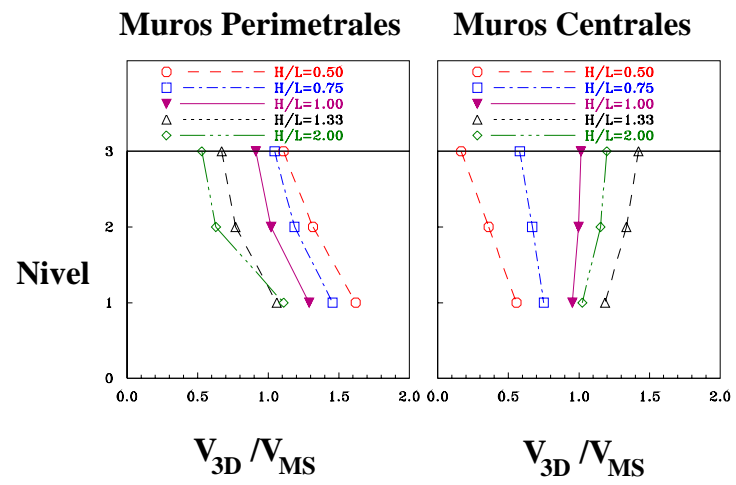

b) Modelos asimétricos

Figura 6. Cortante normalizado $\mathrm{V}_{3 \mathrm{D}} / \mathrm{V}_{\mathrm{MS}}$ para estructuras con distribuciones simétricas y asimétricas de muros en planta (Tena, Pérez y Cano 2002)

\section{REVISIÓN Y PROPUESTA DE FACTORES DE ÁREA EFECTIVA}

Conforme a los estudios iniciales, el primer paso para mejorar las estimaciones de las fuerzas cortantes obtenidas con el método simplificado consiste en mejorar los factores de área efectiva vigentes en las NTCM-2004, que funcionan como factores de ajuste, como se mencionó anteriormente.

Para ello, se realizó un extenso estudio paramétrico en modelos de tres y cinco niveles similares a los edificios ilustrados en la figura 5a (Cano 2005, Cano y Tena 2005) donde: (1) se determinaron las imprecisiones de las ecuaciones 5 y 6 con respecto a análisis estáticos tridimensionales rigurosos, (2) se determinó la forma general que las ecuaciones para los $F_{A E}$ deberían tener después de obtener, a partir de una condensación estática, la matriz de rigidez lateral teórica de muros idealizados como columnas anchas equivalentes y, (3) se determinaron factores de área efectiva para tres distintos niveles de desempeño estructural: a) comportamiento totalmente elástico, correspondiente a un estado límite de servicio (figura 7), b) agrietamiento de todos los muros en los niveles inferiores, correspondiente al estado límite de prevención de colapso de muros de mampostería confinada (figura 8) y, c) agrietamiento de todos los muros en todos los niveles, correspondiente a un estado límite de prevención de colapso para mampostería no confinada ni reforzada (figura 9).

En el estudio paramétrico de referencia se consideró la misma distribución simétrica en planta (figura 5a). Se consideraron cinco casos de estudio diferentes para cada elevación ( 3 o 5 niveles) y para cada uno de los tres distintos niveles de desempeño considerados (elástico, parcialmente agrietado y 
Revisión y actualización del método simplificado de análisis de estructuras de mampostería de los reglamentos de ...

totalmente agrietado). En cada uno de los casos en estudio se mantuvo fija la relación de aspecto del muro central, y se varió la relación de aspecto de los muros laterales. A los muros centrales se les asignó una relación de aspecto $H / L$ desde 0.50 hasta 2.50 , a intervalos de 0.5 , por lo que los casos de estudio se denotan como: $H / L=0.5, H / L=1, H / L=1.5, H / L=2$ y $H / L=2.5$. Para cada uno de estos casos de estudio, se dieron diferentes valores para la relación de aspecto de los muros laterales, desde $H / L=0.42$ hasta $H / L=2.5$. Se seleccionó este intervalo de valores para la relación $H / L$ del muro central por considerar que dentro de este intervalo caen la mayoría de muros que se construyen en la realidad, desde muros muy robustos, con $H / L<1$, hasta muros esbeltos, con $H / L>1.5$. Las propiedades de los muros se describen con detalle en Cano (2005).
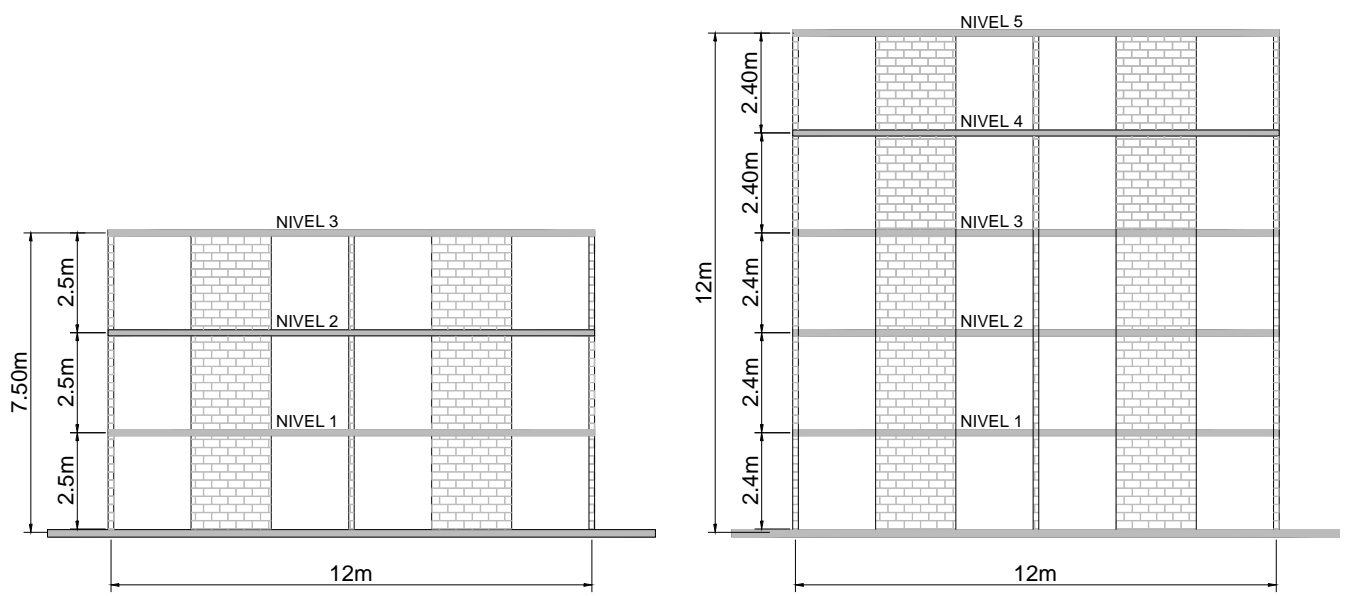

Figura 7. Elevación de los muros de la planta mostrada en la figura 5a en la dirección X para comportamiento totalmente elástico
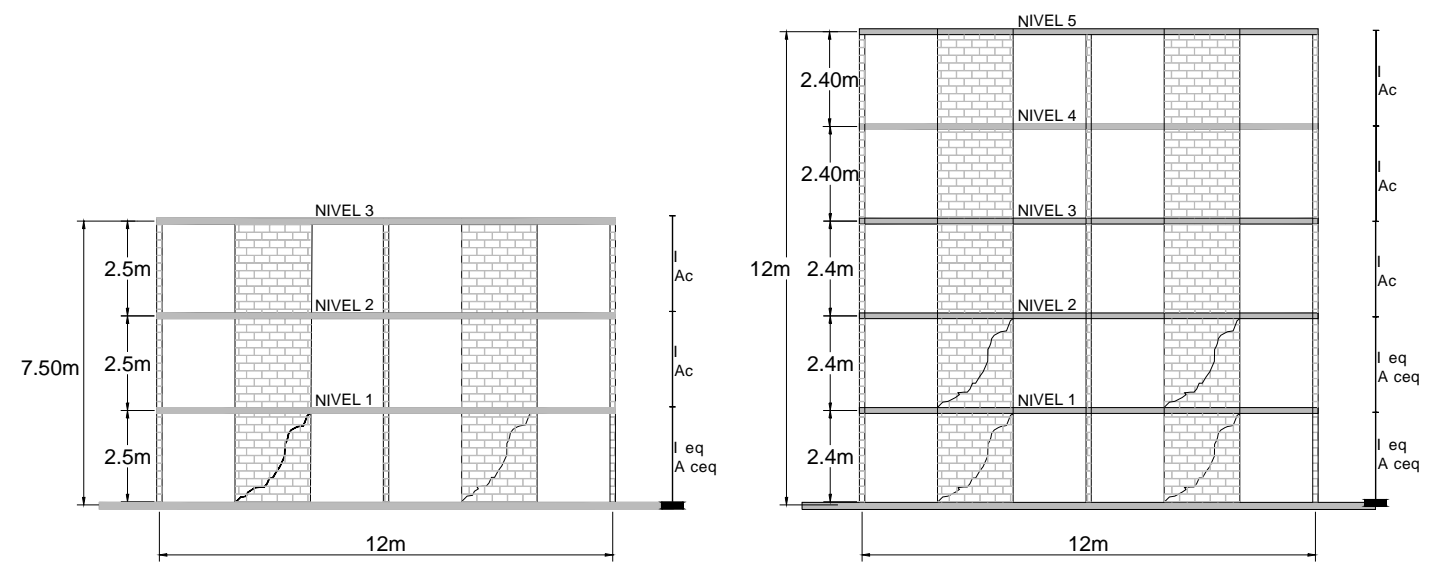

Figura 8. Elevación de los muros de la planta mostrada en la figura 5a en la dirección X para el caso donde existe agrietamiento de todos los muros de los niveles inferiores

La densidad de muros, $\mathrm{D}_{\mathrm{M}}$, definida como el cociente de la sumatoria del área gruesa de todos los muros en la dirección de análisis con respecto al área de la planta y expresada en porcentaje, varió entre $0.87 \%$ cuando todos los muros son esbeltos $(H / L=2.5)$ y $4.93 \%$ cuando todos los muros son robustos $(H / L \leq 0.5)$. La variación de $\mathrm{D}_{\mathrm{M}}$ en función de la relación $H / L$ de los muros centrales, fue la siguiente: a) $2.04 \% \leq \mathrm{D}_{\mathrm{M}} \leq 4.93 \%$ para $H / L=0.5$, b) $1.31 \% \leq \mathrm{D}_{\mathrm{M}} \leq 4.20 \%$ para $H / L=1.0$, c) $1.06 \% \leq \mathrm{D}_{\mathrm{M}} \leq 3.95 \%$ para $H / L=1.5$, d) $0.95 \% \leq \mathrm{D}_{\mathrm{M}} \leq 3.84 \%$ para $H / L=2.0 \mathrm{y}$, e) $0.87 \% \leq \mathrm{D}_{\mathrm{M}} \leq 3.76 \%$ para $H / L=2.5$. 
Conforme a lo expuesto anteriormente, para cada caso de estudio se tiene un total de 43 modelos diferentes. Como se consideran cinco casos de estudio diferentes, en total se obtienen 215 modelos para cada elevación. Al existir modelos de tres y cinco niveles, se tienen 430 modelos para cada desempeño considerado y, al considerarse tres distintos niveles de desempeño, se tienen en total 1290 modelos distintos, a los cuales se les realizaron un análisis estático tridimensional riguroso y varios análisis con el método simplificado: uno utilizando el $F_{A E}$ conforme a la propuesta de las NTCM-2004 y varios más calibrando propuestas de $F_{A E}$ para cada nivel de desempeño considerado.

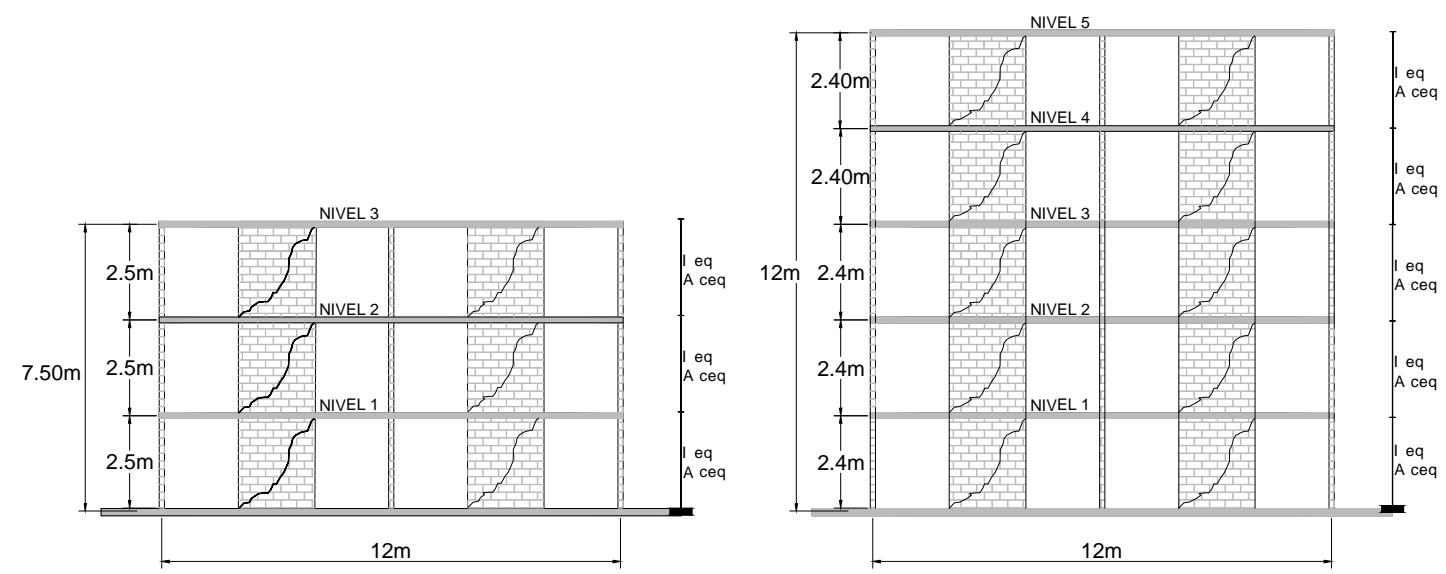

Figura 9. Elevación de los muros de la planta mostrada en la figura 5a en la dirección X para el caso donde existe agrietamiento de todos los muros en todos los niveles

Para modelar la respuesta elástica, los muros se idealizaron como columnas anchas equivalentes (figura $4 \mathrm{~b}$ ) donde se incluyen las deformaciones por cortante conforme a un modelado elástico lineal, homogéneo e isotrópico (por ejemplo, Cano 2005, Cano y Tena 2005, Tena 2007). Cabe señalar que aunque la mampostería no es un material homogéneo y menos isotrópico, se acostumbra modelar de esta manera para comportamiento elástico, debido a las limitaciones que para incluir modelos anisotrópicos han tenido por décadas la inmensa mayoría de los programas de análisis estructural comercial. Es por ello que reglamentos de mampostería como las NTCM-2004 y el ACI 530 permiten modelar de esta manera a los muros de mampostería.

Para considerar el agrietamiento de los muros dentro de límites razonables de daño, se empleó la analogía de la columna ancha agrietada equivalente propuesta por Bazán y Meli (por ejemplo, Bazán y Meli 1998, Tena 2007) con base en el análisis de datos experimentales de muros de mampostería confinada ensayados durante los años $60 \mathrm{~s}$ y 70 s en el Instituto de Ingeniería de la UNAM. Las propiedades de esta columna ancha agrietada equivalente (figura 10) están dadas por las siguientes expresiones:

$$
\begin{aligned}
& I_{e q}=A_{c} \frac{b^{2}}{2} \\
& A_{c e q}=(0.37-0.12 \zeta+0.023 \lambda)\left(A_{m}+2 A_{c}\right) \\
& \zeta=\frac{b}{H} \\
& \lambda=\frac{E_{c} A_{c}}{G_{m} A_{m}}
\end{aligned}
$$


donde $A_{c}$ es el área de la sección transversal de cada castillo (o elemento vertical confinante), $A_{m}$ es el área neta de la sección transversal del muro, ambas sin transformarse, $E_{c}$ es el módulo de elasticidad de los elementos de confinamiento o del marco y $G_{m}$ es el módulo de cortante del muro. Estas expresiones se desarrollaron para $G_{m}=0.4 E_{m}$, donde $E_{m}$ es el módulo de elasticidad del muro. Las expresiones 9 y 10 son válidas para el siguiente intervalo de las relaciones de aspecto $\zeta$ y de rigideces relativas axiales de los castillos (columnas) con respecto al muro $\lambda$ (Bazán y Meli 1998):

$$
\begin{aligned}
& 0.75 \leq \zeta \leq 2.5 \\
& 0.9 \leq \lambda \leq 11
\end{aligned}
$$

Cabe señalar que la columna ancha agrietada equivalente propuesta por Bazán y Meli ha sido revisada con los resultados de ensayes experimentales de muros de mampostería confinada hechos en el Cenapred en la década de los años noventa (por ejemplo, Flores y Alcocer 2001) y de muros de mampostería combinada y confinada hechos en la Universidad Autónoma Metropolitana (Tena-Colunga et al. 2009), donde se han obtenido correlaciones razonables para el agrietamiento asociado al estado límite de diseño conforme a las NTCM-2004 (2004).

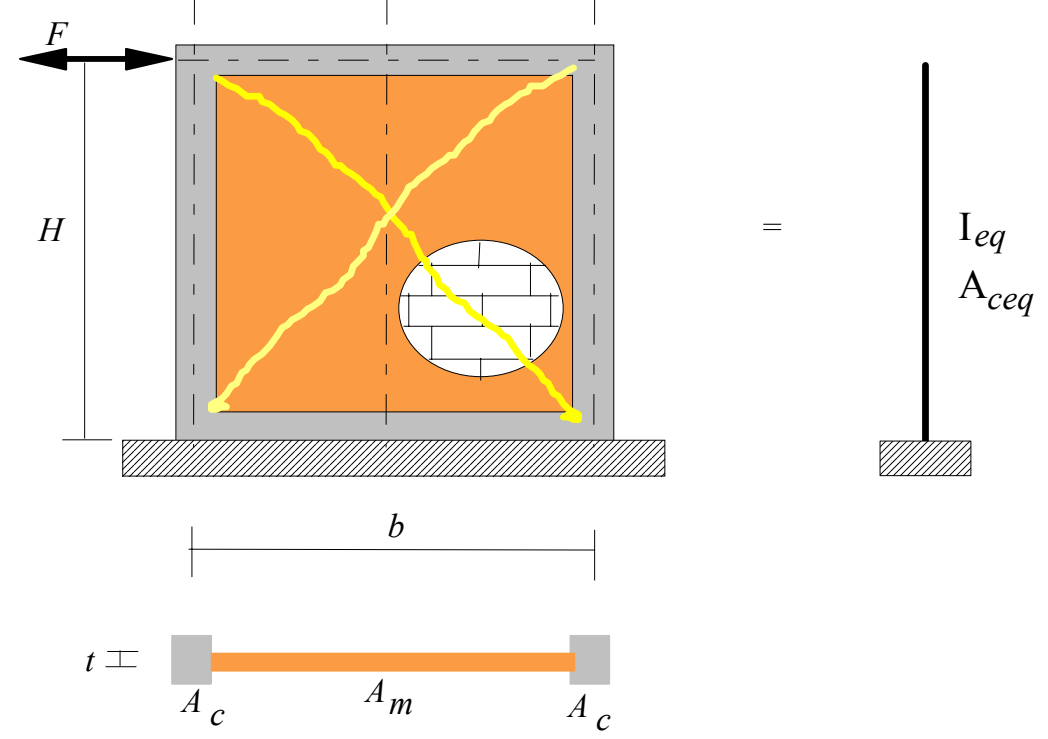

Figura 10. Idealización de un muro confinado agrietado como columna ancha equivalente

\section{Revisión de los factores de área efectiva de las NTCM-2004}

Para revisar la eficiencia de la estimación de las fuerzas cortantes de diseño obtenidas con el método simplificado $\left(\mathrm{V}_{\mathrm{MS}}\right)$ se normalizaron éstas con respecto a las obtenidas conforme a un análisis estático tridimensional riguroso $\left(\mathrm{V}_{3 \mathrm{D}}\right)$ empleando el método de Damy (por ejemplo, Damy 1986, Damy y Alcocer, Tena 2007) que para fines de este estudio se consideran como los más representativos o exactos. Los resultados obtenidos se expresan gráficamente mediante el cociente $\mathrm{V}_{3 \mathrm{D}} / \mathrm{V}_{\mathrm{MS}}$. Por ello, cuando el 
cociente $\mathrm{V}_{3 \mathrm{D}} / \mathrm{V}_{\mathrm{MS}}=1.0$, se tiene una estimación perfecta, mientras que cuando $\mathrm{V}_{3 \mathrm{D}} / \mathrm{V}_{\mathrm{MS}}<1.0$ se tiene una estimación conservadora con el método simplificado y cuando $\mathrm{V}_{3 \mathrm{D}} / \mathrm{V}_{\mathrm{MS}}>1.0$ se tiene una estimación no conservadora con el método simplificado y constituiría, por tanto, los casos críticos.

En la figura 11 se presentan los cocientes $\mathrm{V}_{3 \mathrm{D}} / \mathrm{V}_{\mathrm{MS}}$ para los modelos de tres niveles cuando la respuesta de todos los muros es elástica (figura 7) y se consideran los factores de área efectiva de las NTCM-2004 (ecuaciones 5 y 6) para los casos donde los muros centrales tienen relaciones de aspecto $H / L=0.5,1$ y 2.5 . En el eje de las abcisas se indica la relación de aspecto $(H / L)$ de los muros perimetrales y en el eje de las ordenadas los cocientes $\mathrm{V}_{3 \mathrm{D}} / \mathrm{V}_{\mathrm{MS}}$. La línea continua con rombos identifica a los cocientes obtenidos para el primer nivel (N1) o planta baja, que normalmente es el crítico para fines de diseño. Se observa de la figura 11 que los factores de área efectiva propuestos por las NTCM-2004 no son lo suficientemente adecuados para obtener buenas aproximaciones, particularmente para relaciones de aspecto $H / L \leq 1$ para los muros centrales (figuras 11a y 11b) y que sólo se obtienen mejores aproximaciones a medida que dominan los muros esbeltos (figura 11c). De hecho, de estas figuras se observa que las estimaciones sólo son exactas cuando la relación $H / L$ de todos los muros (centrales y perimetrales) son iguales y tienden a ser poco conservadoras cuando la relación de aspecto de los muros del perímetro son menores a la relación de aspecto de los muros centrales, es decir, $(H / L)_{\text {muros perimetrales }}<$

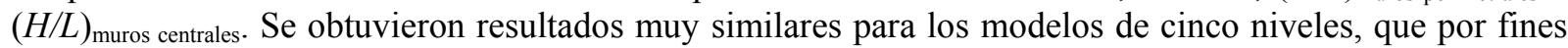
de espacio no se presentan (Cano 2005).

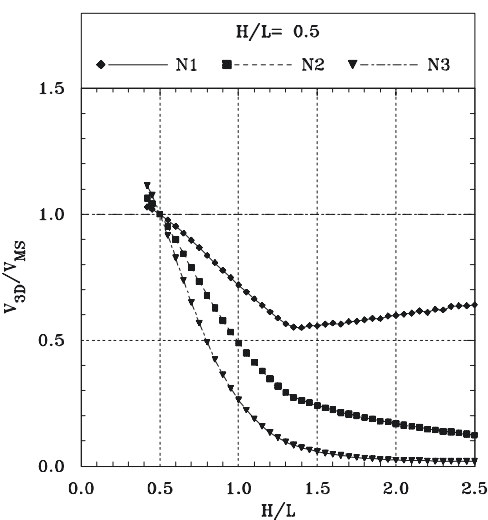

a) $H / L=0.5$ para muros centrales

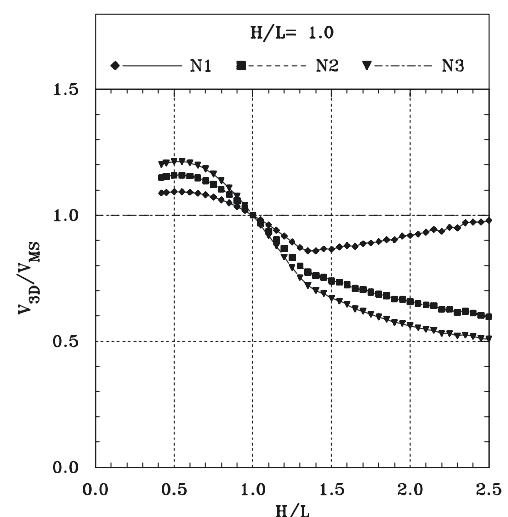

b) $H / L=1.0$ para muros centrales

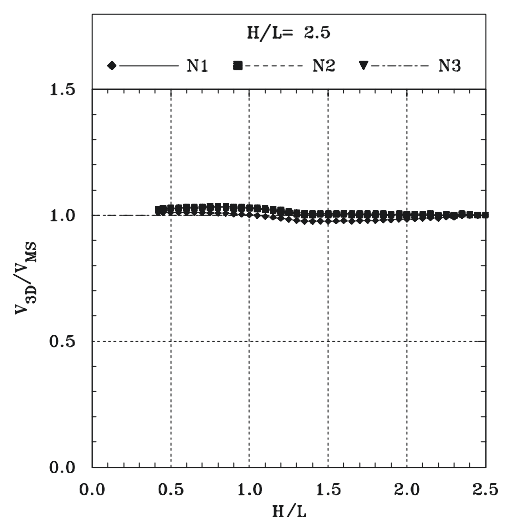

c) $H / L=2.5$ para muros centrales

Figura 11. Cocientes $\mathrm{V}_{3 \mathrm{D}} / \mathrm{V}_{\mathrm{MS}}$ para edificios simétricos de tres pisos con muros con comportamiento elástico utilizando el factor de área efectiva $F_{A E}$ de las NTCM-2004

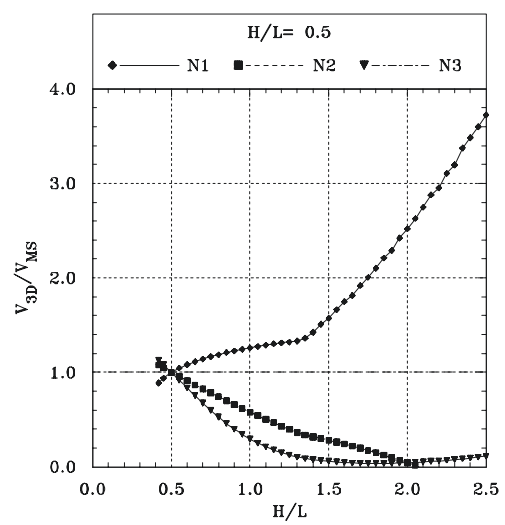

a) $H / L=0.5$ para muros centrales

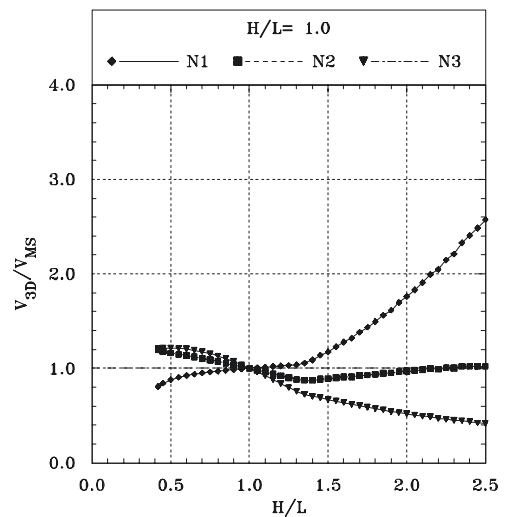

b) $H / L=1.0$ para muros centrales

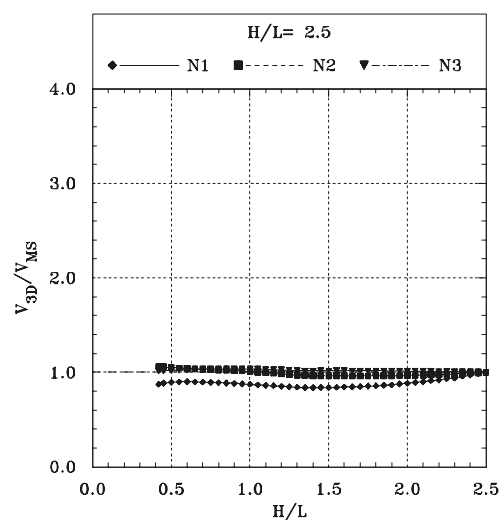

c) $H / L=2.5$ para muros centrales 
Revisión y actualización del método simplificado de análisis de estructuras de mampostería de los reglamentos de ...

Figura 12. Cocientes $\mathrm{V}_{3 \mathrm{D}} / \mathrm{V}_{\mathrm{MS}}$ para edificios simétricos de tres pisos con muros agrietados sólo en los niveles inferiores utilizando el factor de área efectiva $F_{A E}$ de las NTCM-2004

En la figura 12 se presentan los cocientes $\mathrm{V}_{3 \mathrm{D}} / \mathrm{V}_{\mathrm{MS}}$ para los modelos de tres niveles cuando todos los muros del primer nivel están agrietados (figura 8) y se consideran los factores de área efectiva de las NTCM-2004 (ecuaciones 5 y 6) para los casos donde los muros centrales tienen relaciones de aspecto $H / L=0.5,1$ y 2.5 . En este caso, los factores de área efectiva propuestos por las NTCM-2004 son completamente inadecuados para obtener buenas aproximaciones para los muros del primer nivel (N1), obteniéndose aproximaciones poco conservadoras particularmente para relaciones de aspecto $H / L \leq 1$ para los muros centrales (figuras 12a y 12b) y que sólo se obtienen aproximaciones conservadoras a medida que dominan los muros esbeltos (figura 12c). De hecho, de estas figuras se observa nuevamente que las estimaciones sólo son exactas cuando la relación $H / L$ de todos los muros (centrales y perimetrales) son iguales pero, en este caso, al contrario de lo presentado para comportamiento totalmente elástico, las aproximaciones tienden a ser muy poco conservadoras cuando las relaciones de aspecto de los muros del perímetro son mayores a la relación de aspecto de los muros centrales, es decir, $(H / L)_{\text {muros perimetrales }}>$

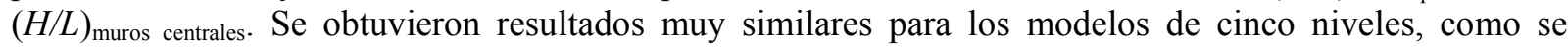
presenta en Cano (2005).

En la figura 13 se presentan los cocientes $\mathrm{V}_{3 \mathrm{D}} / \mathrm{V}_{\mathrm{MS}}$ para los modelos de tres niveles cuando todos los muros de todos los niveles están agrietados (figura 9) y se consideran los factores de área efectiva de las NTCM-2004 (ecuaciones 5 y 6) para los casos donde los muros centrales tienen relaciones de aspecto $H / L=0.5,1$ y 2.5 . En este caso se pueden hacer observaciones muy similares a las realizadas previamente para el caso donde se considera exclusivamente el agrietamiento de todos los muros de los niveles inferiores (figuras 8 y 12).

Por lo tanto, conforme a los resultados del estudio paramétrico que se ha resumido brevemente en esta sección, se concluyó que era necesario mejorar los factores de área efectiva del método simplificado para cada uno de los niveles de desempeño estructural considerados, sobre todo cuando se considera el agrietamiento de todos los muros en los niveles inferiores (figura 8), ya que este desempeño estructural corresponde al estado límite de prevención de colapso de muros de mampostería confinada contemplado por las NTCM-2004, avalado por los resultados obtenidos en ensayes experimentales de modelos a escala natural ante carga cíclica en muro de reacción (Ruiz 1995, Ruiz y Alcocer 1998) como en ensayes de modelos a escala en mesa vibradora (Arias et al. 2004).

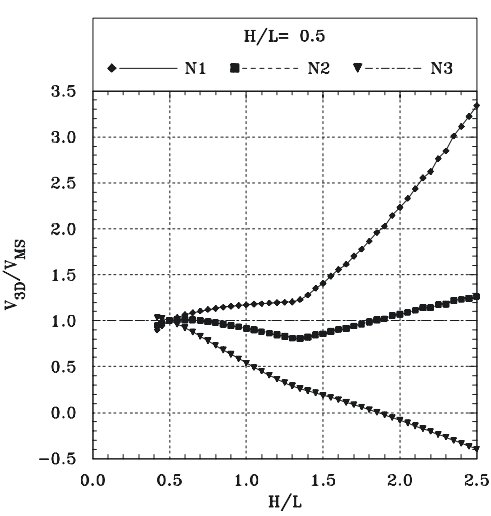

a) $H / L=0.5$ para muros centrales

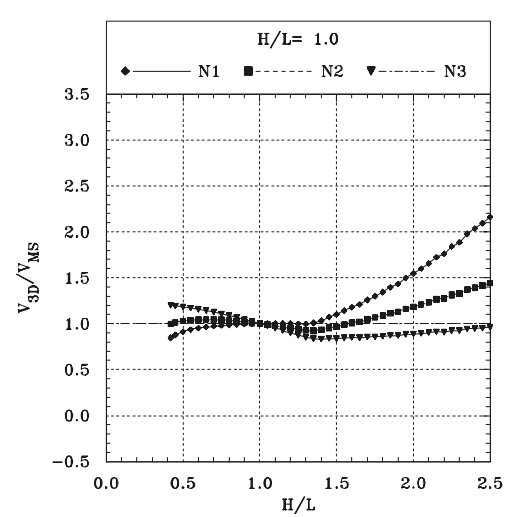

b) $H / L=1.0$ para muros centrales

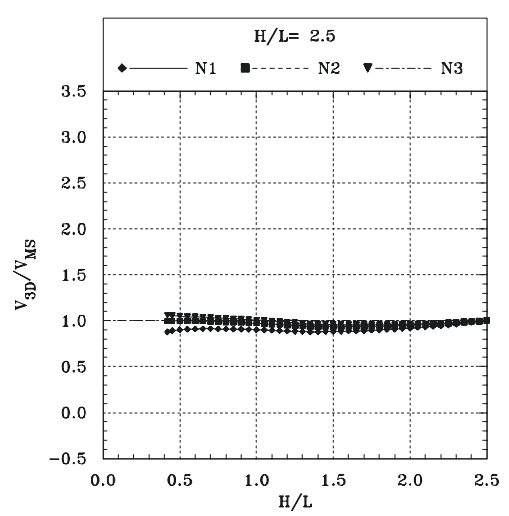

c) $H / L=2.5$ para muros centrales

Figura 13. Cocientes $V_{3 D} / V_{M S}$ para edificios simétricos de tres pisos con muros agrietados en toda la elevación utilizando el factor de área efectiva $F_{A E}$ de las NTCM-2004 


\section{Propuesta de nuevos factores de área efectiva para respuesta elástica}

El primer paso para poder proponer factores de área efectiva que lideren a mejores aproximaciones del método simplificado con respecto a un análisis tridimensional riguroso fue racionalizar a partir de la teoría del análisis estructural elástico lineal cómo deberían lucir estas ecuaciones si se considera que los muros pueden ser idealizados como columnas anchas equivalentes donde se incluyen las deformaciones por cortante (figura 4). Los detalles de este desarrollo se presentan en Cano (2005) y Cano y Tena (2005) y se resumen a continuación.

Si se realiza una condensación estática de la matriz de rigidez global de los muros en elevación presentados en la figura 4 (Cano y Tena 2005), se obtiene que los coeficientes de rigidez $k_{i j}$ de la matriz de rigidez lateral resultante son de la forma:

$$
\left[k_{i j \Delta}\right]=\frac{a E t}{1+3 \frac{L^{2}}{H^{2}}}\left[1-\frac{b+c \frac{L^{2}}{H^{2}}}{d+e \frac{L^{2}}{H^{2}}+f \frac{L^{4}}{H^{4}}}\right]
$$

donde $E, t, L$ y $h$ son respectivamente el módulo de elasticidad, el espesor, la longitud y la altura del muro y $a, b, c, d, e$ y $f$ son coeficientes numéricos que dependen de la condensación estática de la matriz de rigidez global.

Se pueden hacer las siguientes observaciones de los coeficientes de rigidez de la matriz de rigidez lateral dados por la ecuación 15: (1) existen formas polinomiales en función de la relación de aspecto $H / L$ del muro y, (2) si se desea mejorar las aproximaciones del método simplificado para la estimaciones de los cortantes de los muros en todos los niveles, se requeriría de matrices de corrección con distintos factores de área efectiva en su diagonal principal y términos fuera de esta diagonal. Dado que implantar la última observación sería impráctico (en ese caso es mejor hacer un análisis riguroso), se tomó la decisión que dado que no se pueden mejorar las aproximaciones obtenidas con el método simplificado para todos los niveles, entonces los factores de área efectiva que se propusieran deberían mejorar la aproximación obtenida para los muros del primer nivel (N1), dado que el primer nivel o nivel de planta baja es comúnmente el nivel crítico para el diseño por sismo de estructuras de baja altura con base en muros.

A partir de la observación de la ecuación 15 se puede concluir que una forma polinomial relativamente simple se puede utilizar para mejorar las estimaciones de la fuerza cortante del método simplificado $\left(\mathrm{V}_{\mathrm{MS}}\right)$ de manera que coincidan de mejor manera con un análisis estático tridimensional riguroso $\left(\mathrm{V}_{3 \mathrm{D}}\right)$, por lo tanto:

$$
V_{3 D}=\left[c_{1}+c_{2} \frac{H}{L}+c_{3}\left(\frac{H}{L}\right)^{2}\right] V_{M S}=F_{A E} V_{M S}
$$

Se utilizó el siguiente procedimiento para poder proponer nuevos factores de área efectiva para comportamiento elástico $\left(F_{A E}\right)$ : (1) Las fuerzas cortantes obtenidas para el primer nivel con el análisis estático tridimensional $\left(\mathrm{V}_{3 \mathrm{D}}\right)$ se normalizaron con las obtenidas con el método simplificado sin corregir por un factor de área efectiva (o dicho de otra manera, tomando $F_{A E}=1$ ), para visualizar la forma de estas 
Revisión y actualización del método simplificado de análisis de estructuras de mampostería de los reglamentos de ...

curvas, (2) Con base en lo anterior, a partir de un análisis de regresión se propusieron ecuaciones polinomiales similares a la dada por la ecuación 16 , para posteriormente evaluar su validez y exactitud para el primer nivel. Las ecuaciones finales obtenidas conforme a este procedimiento son las siguientes:

$$
\begin{array}{lll}
F_{A E}=1.5+\frac{H}{L}-1.5\left(\frac{H}{L}\right)^{2} & \text { si } & 0.4 \leq \frac{H}{L} \leq 1.0 \\
F_{A E}=2.2-1.5 \frac{H}{L}+0.3\left(\frac{H}{L}\right)^{2} & \text { si } & 1.0<\frac{H}{L} \leq 2.5
\end{array}
$$

En la figura 14 se presentan los cocientes $\mathrm{V}_{3 \mathrm{D}} / \mathrm{V}_{\mathrm{MS}}$ para los modelos de tres niveles cuando la respuesta de todos los muros es elástica (figura 7) y se consideran los factores de área efectiva propuestos (ecuaciones 17 y 18) para los casos donde los muros centrales tienen relaciones de aspecto $H / L=0.5,1$ y 2.5, donde se puede corroborar que la estimación de las fuerzas cortantes obtenida con el método simplificado empleando estos nuevos $F_{A E}$ mejora notablemente con respecto a la obtenida con los factores de área efectiva de las NTCM-2004 vigentes (figura 11).

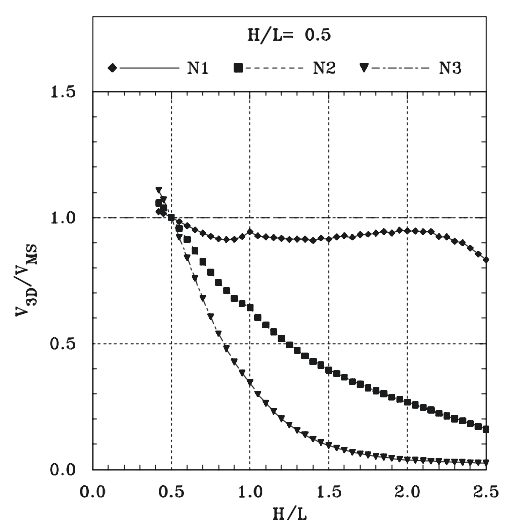

a) $H / L=0.5$ para muros centrales

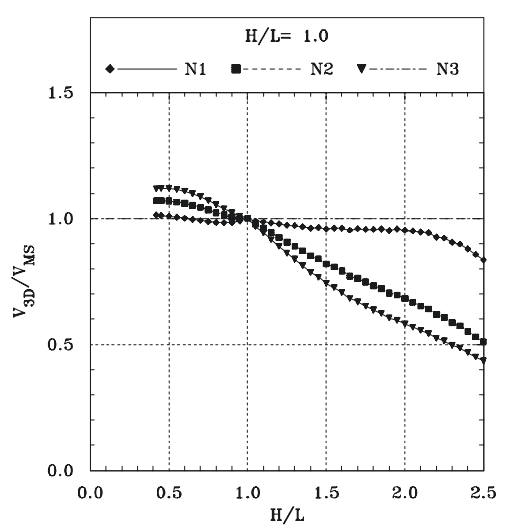

b) $H / L=1.0$ para muros centrales

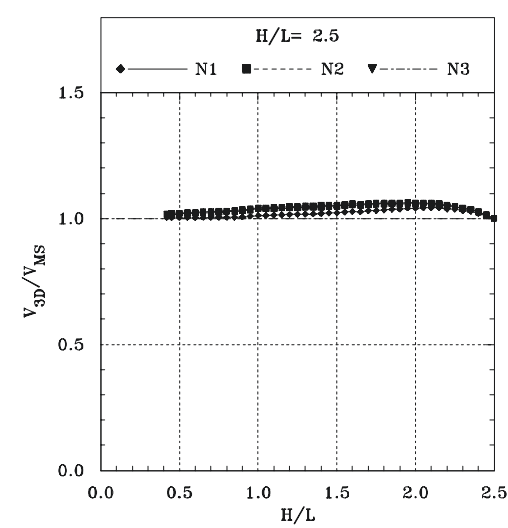

c) $H / L=2.5$ para muros centrales

Figura 14. Cocientes $\mathrm{V}_{3 \mathrm{D}} / \mathrm{V}_{\mathrm{MS}}$ para edificios simétricos de tres pisos con muros con comportamiento elástico utilizando el factor de área efectiva $F_{A E}$ propuesto (ecuaciones 17 y 18)

\section{Propuesta de un nuevo factor de área efectiva para muros agrietados sólo en los niveles inferiores}

Este comportamiento estructural que considera el agrietamiento de todos los muros en los niveles inferiores se ilustra en la figura $8 \mathrm{y}$, como se comentó anteriormente, este desempeño estructural corresponde al estado límite de prevención de colapso de muros de mampostería confinada contemplado por las NTCM-2004.

El procedimiento utilizado para obtener el factor de área efectiva para esta condición es similar al descrito anteriormente para muros con comportamiento elástico, con la diferencia que, en este caso, en los análisis estáticos tridimensionales rigurosos los muros que se consideraron agrietados fueron modelados con la columna ancha agrietada equivalente propuesta por Bazán y Meli (ecuaciones 9 a 14), mientras que los muros que permanecen elásticos se idealizaron como columnas anchas equivalentes que incluyen las deformaciones por cortante conforme a la teoría de vigas de Bernoulli-Euler. 
La ecuación obtenida para este factor de área efectiva para edificios donde se agrietan todos los muros de los niveles inferiores, o "muros parcialmente agrietados" en elevación $\left(F_{A E P A}\right)$, es la siguiente:

$$
F_{A E P A}=0.6+0.6\left(\frac{H}{L}\right)-0.3\left(\frac{H}{L}\right)^{2}+0.05\left(\frac{H}{L}\right)^{3} \quad \text { si } \quad 0.4 \leq \frac{H}{L} \leq 2.5
$$

En la figura 15 se presentan los cocientes $\mathrm{V}_{3 \mathrm{D}} / \mathrm{V}_{\mathrm{MS}}$ para los modelos de tres niveles cuando se agrietan todos los muros del primer nivel (figura 8) y se considera el factor de área efectiva propuesto (ecuación 19) para los casos donde los muros centrales tienen relaciones de aspecto $H / L=0.5,1$ y 2.5 . De esta figura se puede corroborar que, para este complicado estado de desempeño, la estimación de las fuerzas cortantes obtenida con el método simplificado empleando este nuevo $F_{A E P A}$ mejora notablemente con respecto a la obtenida con los factores de área efectiva de las NTCM-2004 vigentes (figura 12). De hecho, las aproximaciones son notablemente buenas para relaciones de aspecto de los muros centrales $H / L \geq 1$ para todo el intervalo de relaciones $H / L$ considerado para los muros perimetrales $(0.5 \leq H / L \leq 2.5)$, como se observa de las figuras $15 \mathrm{~b}$ y $15 \mathrm{c}$. Sin embargo, las estimaciones son ligeramente no conservadoras (hasta un 20\%) cuando la relación de aspecto de los muros centrales es $H / L=0.5$ (figura $15 \mathrm{a})$.

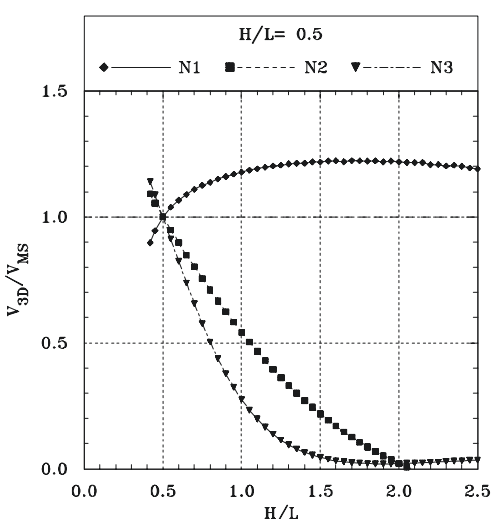

a) $H / L=0.5$ para muros centrales

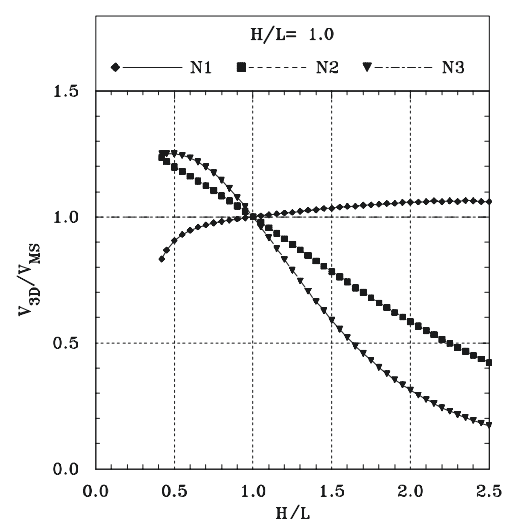

b) $H / L=1.0$ para muros centrales

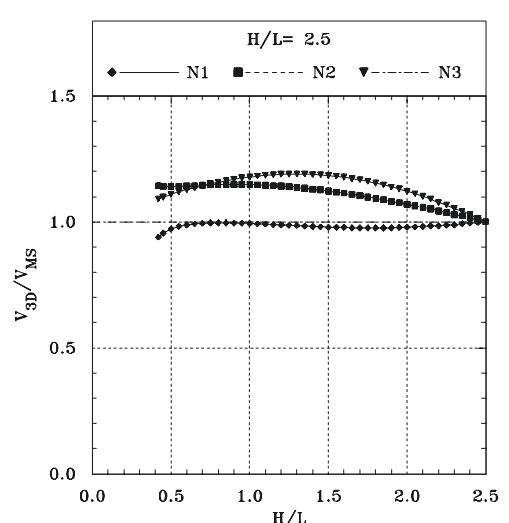

c) $H / L=2.5$ para muros centrales

Figura 15. Cocientes $\mathrm{V}_{3 \mathrm{D}} / \mathrm{V}_{\mathrm{MS}}$ para edificios simétricos de tres pisos con muros agrietados sólo en los niveles inferiores utilizando el factor de área efectiva $F_{A E P A}$ propuesto (ecuación 19)

\section{Propuesta de un nuevo factor de área efectiva para muros agrietados en todos los niveles}

Este comportamiento estructural que considera el agrietamiento de todos los muros en todos los niveles inferiores se ilustra en la figura $9 \mathrm{y}$, como se comentó anteriormente, este desempeño estructural corresponde al observado en muros de mampostería no confinada ni reforzada (o hecho de manera deficiente) durante sismos intensos.

El procedimiento utilizado para obtener el factor de área efectiva para esta condición es similar al descrito en secciones previas. En este caso, en los análisis estáticos tridimensionales rigurosos todos los muros se consideraron agrietados y fueron modelados con la columna ancha agrietada equivalente propuesta por Bazán y Meli (ecuaciones 9 a 14).

La ecuación obtenida para este factor de área efectiva para edificios donde se agrietan todos los muros en todos los niveles, o "muros totalmente agrietados" en elevación $\left(F_{A E T A}\right)$, es la siguiente: 


$$
F_{A E T A}=1+1.1\left(\frac{H}{L}\right)-0.6\left(\frac{H}{L}\right)^{2}+0.1\left(\frac{H}{L}\right)^{3} \quad \text { si } 0.4 \leq \frac{H}{L} \leq 2.5
$$

En la figura 16 se presentan los cocientes $\mathrm{V}_{3 \mathrm{D}} / \mathrm{V}_{\mathrm{MS}}$ para los modelos de tres niveles cuando se agrietan todos los muros (figura 9) y se considera el factor de área efectiva propuesto (ecuación 20) para los casos donde los muros centrales tienen relaciones de aspecto $H / L=0.5,1$ y 2.5 . De esta figura se pueden hacer las mismas observaciones que se hicieron para el caso "parcialmente agrietado": (1) la estimación de las fuerzas cortantes obtenida con el método simplificado empleando este nuevo $F_{A E T A}$ mejora notablemente con respecto a la obtenida con los factores de área efectiva de las NTCM-2004 vigentes (figura 13), (2) las aproximaciones son notablemente buenas para relaciones de aspecto de los muros centrales $H / L \geq 1$ para todo el intervalo de relaciones $H / L$ considerado para los muros perimetrales (figuras $16 \mathrm{~b}$ y $16 \mathrm{c}$ ) y, 3 ) sin embargo, las estimaciones son ligeramente no conservadoras (10\%) cuando la relación de aspecto de los muros centrales es $H / L=0.5$ (figura 16a).

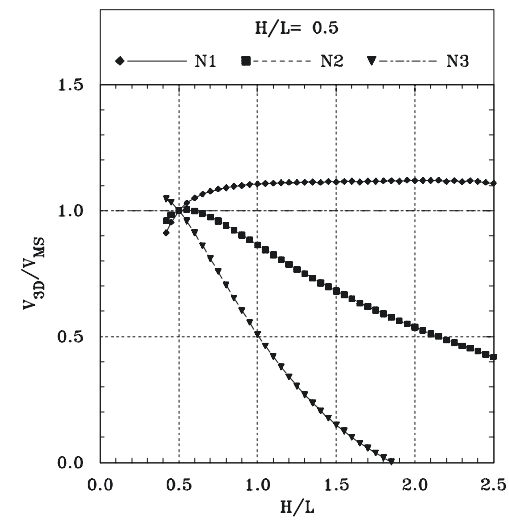

a) $H / L=0.5$ para muros centrales

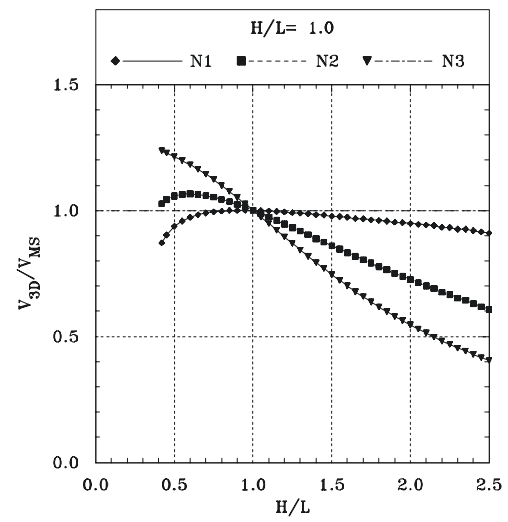

b) $H / L=1.0$ para muros centrales

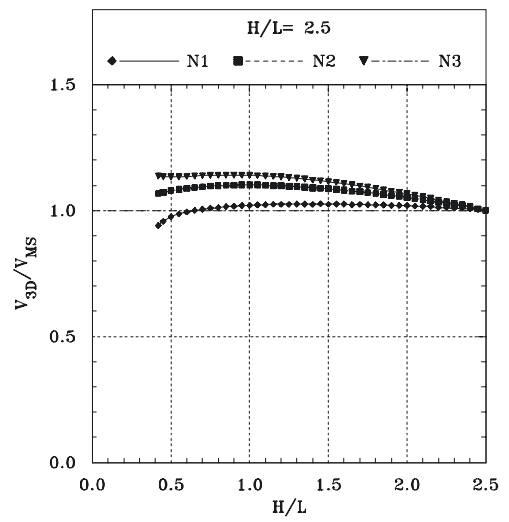

c) $H / L=2.5$ para muros centrales

Figura 16. Cocientes $\mathrm{V}_{3 \mathrm{D}} / \mathrm{V}_{\mathrm{MS}}$ para edificios simétricos de tres pisos con muros agrietados en toda la elevación utilizando el factor de área efectiva $F_{A E T A}$ propuesto (ecuación 20)

\section{EVALUACIÓN DE LA EXCENTRICIDAD ESTÁTICA LÍMITE}

Se realizó un extenso estudio paramétrico donde se valoró el valor límite de la excentricidad estática en planta $\left(e_{s}\right)$ que debe permitirse al método simplificado de análisis para los niveles de desempeño estructural descritos y estudiados previamente, como se reporta con detalle en Tena y López (2006) y López (2007). El estudio toma como fundamento que las subestimaciones de las fuerzas cortantes obtenidas con el método simplificado para los muros críticos se encuentren dentro de límites razonables al compararse con análisis tridimensionales rigurosos, lo que permita garantizar diseños seguros de estructuras de mampostería.

Para el estudio se consideraron edificios de mampostería de tres y cinco niveles con altura típica de entrepiso igual a $2.5 \mathrm{~m}$ para todos los niveles, por lo que la altura total de los edificios de tres y cinco niveles es $7.5 \mathrm{~m}$ y $12.5 \mathrm{~m}$ respectivamente. La planta de los edificios se muestra en la figura 17 , donde se ilustra la disposición de los muros, seis en dirección X y cinco en dirección $\mathrm{Y}$. El uso del edificio es 
habitacional. En todos los casos los muros de mampostería son de tabique rojo recocido de $12.5 \mathrm{~cm}$ de espesor, con módulo de elasticidad $E=24,000 \mathrm{~kg} / \mathrm{cm}^{2}$ y relación de Poisson $v=0.25$. Ambos edificios cumplen con todos los requisitos del método simplificado, entre ellos el que establece que la altura total del edificio debe ser menor o igual a $13 \mathrm{~m}$.

El estudio paramétrico consideró lo siguiente:

1. Las excentricidades estáticas en planta $\left(e_{s}\right)$ se definieron a partir de la posición excéntrica del centro de masa en la dirección del eje $Y$ en la planta tipo (figura 17), ubicándolo de acuerdo a la excentricidad estática deseada. En la dirección $X$, la posición del centro de masa coincide con el centroide de la planta tipo (figura 17). El estudio consideró excentricidades estáticas del $5 \%$, $7.5 \%, 10 \%$ y $20 \%$.

2. De manera similar al estudio previo realizado por Cano (2005), los cuatro muros perimetrales ("MP", muros tipo 1 de la figura 17) en la dirección X varían su relación de aspecto $H / L$ entre 0.5 y 2.5 pero en intervalos de 0.1 , mientras que a los dos muros centrales ("MC", muros tipo 2 de la figura 17) se les asignó una relación de aspecto $H / L$ entre 0.5 y 2.5 a intervalos de 0.5 . Los muros en la dirección Y permanecen invariantes, con una relación de aspecto $H / L=1.0$.

3. De acuerdo con la variación considerada en la relación de aspecto de los dos muros centrales, se tienen cinco casos de estudio y cada uno de éstos contiene veintiún modelos, de acuerdo con la variación de la relación de aspecto de los muros perimetrales, por lo que se obtienen 105 modelos en total para cada edificio (tres o cinco niveles) y desempeño estructural considerado: (a) elástico (fig 7, ecs 17 y 18), (b) "parcialmente agrietado" (fig 8, ec 19) y (c) totalmente agrietado (fig 9, ec 20).

4. Por lo tanto, considerando los tres distintos casos de desempeño (en función de los factores de área efectiva), se tiene un total de 315 modelos diferentes por elevación analizada, y tomando en cuenta que se consideran edificios de tres y cinco niveles, se analizaron 630 modelos diferentes para una excentricidad dada. Puesto que se consideraron cuatro distintos valores para las excentricidades estáticas, el estudio paramétrico que se presenta considera un total de 2,520 modelos o edificios distintos que se analizaron tanto con el método simplificado, como con análisis tridimensionales rigurosos, por lo que el total de análisis realizados fue de 5,040.

5. Todos los modelos se analizaron empleando el método simplificado tomando en cuenta los factores de área efectiva propuestos por Cano y Tena (2005), además de análisis estáticos tridimensionales rigurosos. Ambos análisis sólo consideran la acción de las fuerzas sísmicas en la dirección X, como se ilustra en la figura 17.

En este estudio paramétrico también se compararon principalmente las fuerzas cortantes que toman cada muro en planta baja de acuerdo con el método simplificado con respecto a las obtenidas con un método riguroso de análisis tridimensional. Una vez obtenidas las fuerzas cortantes, se normalizaron las fuerzas cortantes obtenidas mediante el método riguroso $\left(\mathrm{V}_{3 \mathrm{D}}\right)$ con respecto a las obtenidas con el método simplificado $\left(\mathrm{V}_{\mathrm{MS}}\right)$.

El estudio se enfocó más a definir el valor límite de la excentricidad estática que debiera permitírsele al método simplificado. Por lo tanto, se propone aceptar que con el método simplificado se obtenga una subestimación máxima de la fuerza cortante del $30 \%-40 \%$, es decir, $\mathrm{V}_{3 \mathrm{D}} / \mathrm{V}_{\mathrm{MS}} \leq 1.4$. Esta propuesta toma en cuenta tres aspectos esencialmente: (a) los muy bajos valores de $v^{*}{ }_{m}$ de las mamposterías mexicanas, donde un $30 \%-40 \%$ de diferencia puede redundar en que se requieran utilizar 
Revisión y actualización del método simplificado de análisis de estructuras de mampostería de los reglamentos de ...

piezas o morteros de pegas distintos o, inclusive, se requiera de refuerzo horizontal, (b) cuando se emplea el método simplificado de análisis, no se toman en cuenta los efectos bidireccionales más que de una manera aproximada en el coeficiente sísmico de diseño propuesto para usar este método y, (c) cuando se usa el método simplificado no se amplifican las fuerzas adicionales por torsión para tomar en cuenta excentricidades accidentales y efectos dinámicos, como sí exigen las NTCS-2004 para cuando se emplean otros métodos de análisis (estático y dinámico).

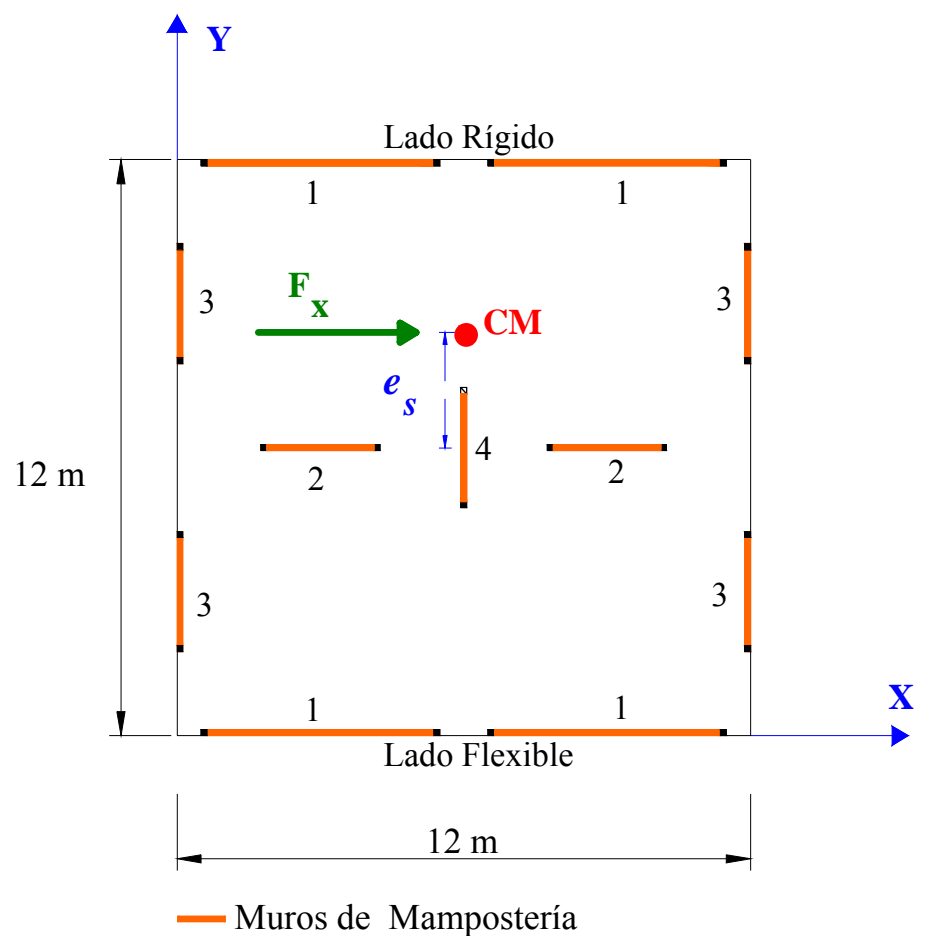

Figura 17. Planta tipo de los modelos empleados para el estudio paramétrico

Los resultados más interesantes con fines prácticos y para su discusión son los que se presentan en las Figuras 18 a 21, que son aquéllos relacionados con el valor de la excentricidad estática $e_{s}$ que debe tolerarse en el método simplificado con fines de diseño para el desempeño estructural en estudio. Cabe señalar que en las figuras 18 a 21 se grafican todos los resultados obtenidos para la excentricidad y factor de área efectiva en estudio. En estas figuras, se emplean símbolos vacíos conectados con líneas continuas para presentar los resultados de los muros centrales (MC), mientras que los símbolos llenos conectados con líneas discontinuas se emplean para identificar los resultados de los muros perimetrales (MP) críticos. Por ejemplo, cuando la relación de aspecto de los muros centrales es $H / L=1$, para el intervalo de relaciones de aspecto $H / L$ considerado para los muros perimetrales, los cuadrados vacíos se emplean para graficar los cocientes $\mathrm{V}_{3 \mathrm{D}} / \mathrm{V}_{\mathrm{MS}}$ para los muros centrales del nivel de planta baja, mientras que los cuadrados llenos se emplean para graficar los cocientes $\mathrm{V}_{3 \mathrm{D}} / \mathrm{V}_{\mathrm{MS}}$ para los muros perimetrales críticos en ese mismo nivel.

El estudio de referencia se presenta de manera sintética en las siguientes secciones. Los detalles se pueden consultar en Tena y López (2006) y López (2007). 


\section{Evaluación para comportamiento totalmente elástico de los muros}

Considérese el caso en que todos los muros se comportan elásticamente (fig 7). Tomando los $F_{A E}$ propuestos (ecs 17 y 18) y conforme a los resultados obtenidos para los muros centrales y perimetrales para todas las excentricidades en estudio (López 2007), aunque aquí sólo se presentan las gráficas para 5\% para los edificios de tres y cinco niveles (figura 18), se pueden hacer las siguientes observaciones.

En términos generales, las aproximaciones obtenidas con el método simplificado utilizando los factores de área efectiva para comportamiento elástico no son razonables aun para una excentricidad estática del 5\%. Cuando los muros centrales son robustos $(H / L \leq 1)$, se subestiman notablemente las fuerzas cortantes de los muros perimetrales (MP) críticos, particularmente para las siguientes relaciones de aspecto de los muros perimetrales: $1.5 \leq H / L \leq 2.5$. Cuando los muros centrales son esbeltos $(H / L \geq 1.5)$, sus fuerzas cortantes correspondientes se subestiman enormemente para siguientes relaciones de aspecto de los muros perimetrales: $0.5 \leq H / L \leq 1.5$.

Por lo tanto y conforme a los resultados obtenidos, se puede concluir que, para fines prácticos, se recomienda emplear el método simplificado cuando todos los muros se comportan elásticamente, correspondiente a un estado límite de servicio, para excentricidades estáticas en planta hasta del cinco porciento $\left(e_{s}=5 \%\right)$ o menores. Sin embargo, en rigor se deberían observar las siguientes limitaciones, tomando en cuenta los resultados mostrados en la figura 18 y que se acepta como razonable que el método simplificado subestime hasta en un $30 \%-40 \%$ la fuerza cortante de diseño $\left(\mathrm{V}_{3 \mathrm{D}} / \mathrm{V}_{\mathrm{MS}} \leq 1.4\right)$. Para este nivel de desempeño, el método simplificado sólo podría utilizarse con confianza cuando las relaciones de aspecto tanto de los muros centrales como de los perimetrales se encuentren en el siguiente intervalo: $1.0 \leq H / L \leq 1.5$, según se observa en la figura 18 .

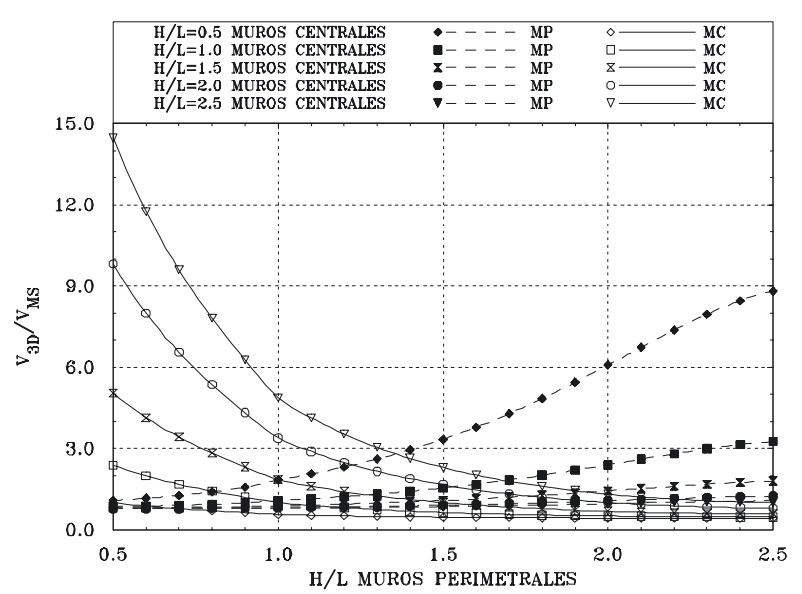

a) Edificios de tres niveles

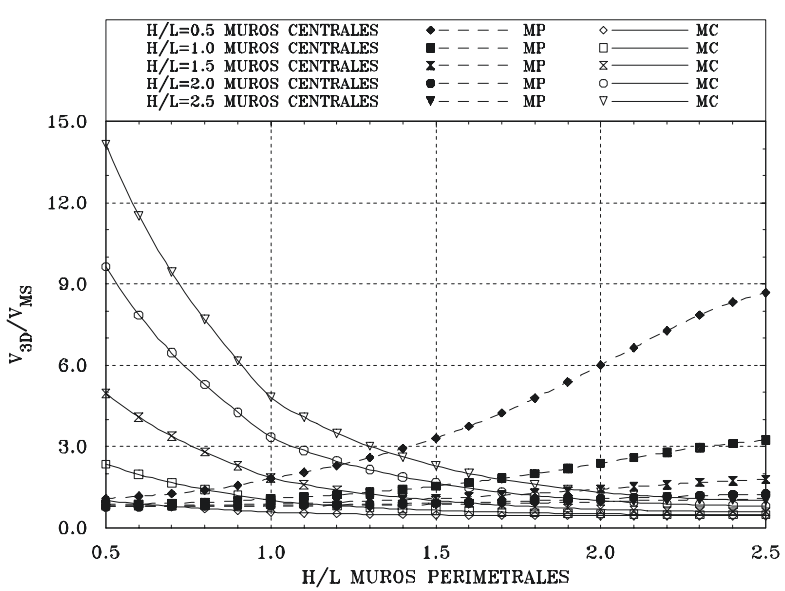

b) Edificios de 5 niveles

Figura 18. Relación de fuerzas cortantes $\mathrm{V}_{3 \mathrm{D}} / \mathrm{V}_{\mathrm{MS}}$ para los muros del primer nivel de los edifícios de la figura 17 cuando $e_{s}=5 \%$, el comportamiento es totalmente elástico y se emplean los factores de área efectiva propuestos (ecs 17 y 18). Las curvas se trazan para las relaciones de aspecto $H / L$ de los muros centrales indicados en las leyendas. 


\section{Evaluación del $\mathbf{F}_{\mathrm{AE}}$ de las NTCM-2004}

Conforme a las NTCM-2004, el estado límite de prevención de colapso de estructuras de mampostería confinada está asociado al agrietamiento de todos los muros en los niveles inferiores (figura 8). Por lo tanto, es para este nivel de desempeño para el cual se debe determinar la excentricidad estática $\left(e_{s}\right)$ que puede permitírsele al método simplificado cuando se emplean los factores de área efectiva propuestos por las NTCM-2004.

Los resultados obtenidos para los edificios de tres y cinco niveles para $e_{s}=5 \%$ se presentan en la figura 19. Se observa que los resultados de los edificios de tres niveles (figura 19a) y de cinco niveles (figura 19b) son algo contrastantes. Conforme a los resultados de los modelos de tres niveles, las aproximaciones obtenidas con el método simplificado cuando $e_{s}=5 \%$ son pobres y del lado de la inseguridad para algunos muros centrales y perimetrales, particularmente: (a) muros perimetrales (MP) con relaciones de aspecto en el intervalo $1.7 \leq H / L \leq 2.5$ cuando las relaciones de aspecto de los muros centrales se encuentran dentro del intervalo $0.5 \leq H / L \leq 1.5 \mathrm{y}$, (b) muros centrales (MC) con relaciones de aspecto en el intervalo $2.0 \leq H / L \leq 2.5$ cuando las relaciones de aspecto de los muros perimetrales están dentro del intervalo $0.5 \leq H / L \leq 1.5$. En contraste, las aproximaciones obtenidas con el método simplificado para los edificios de cinco niveles es razonablemente buena y segura para $e_{s}=5 \%$ para todas las relaciones de aspecto consideradas para los muros centrales y perimetrales, con excepción quizás cuando los muros centrales son muy esbeltos $(H / L=2.5)$ y las relaciones de aspecto de los muros perimetrales se encuentra en el siguiente intervalo: $1.2 \leq H / L \leq 1.6$.

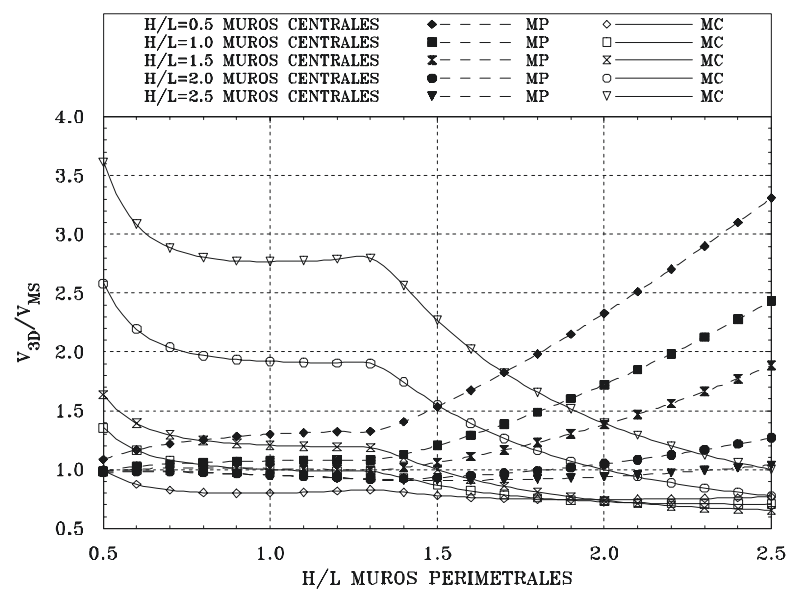

a) Modelos de tres niveles

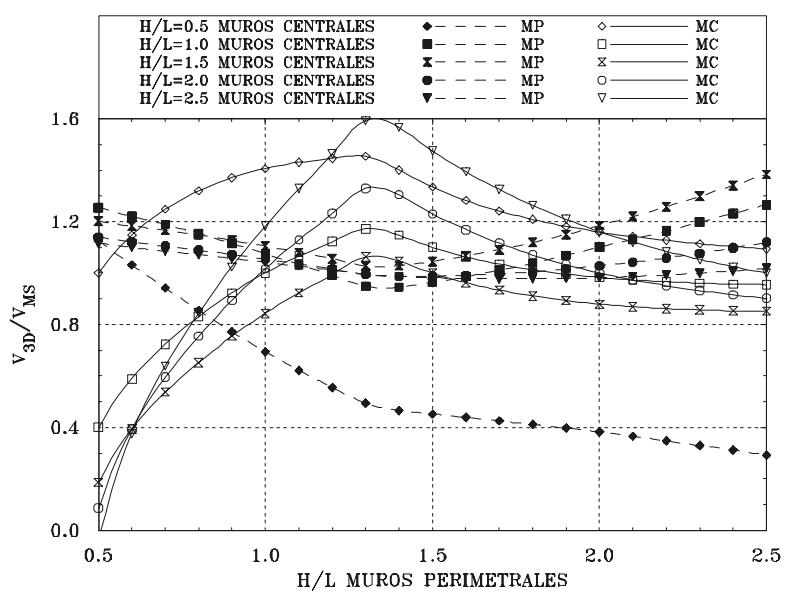

b) Modelos de cinco niveles

Figura 19. Relación de fuerzas cortantes $\mathrm{V}_{3 \mathrm{D}} / \mathrm{V}_{\mathrm{MS}}$ para los muros del primer nivel de los edificios de la figura 17 cuando $e_{s}=5 \%$, el comportamiento es parcialmente agrietado y se emplean los factores de área efectiva de las NTCM-2004. Las curvas se trazan para las relaciones de aspecto $H / L$ de los muros centrales indicados en las leyendas.

Por lo tanto, se puede concluir con base en los resultados obtenidos para los edificios de cinco niveles que, para fines prácticos, se puede permitir una excentricidad estática $e_{s}$ hasta del $5 \%$ para el método simplificado conforme a las NTCM-2004, en lugar de la $e_{s}=10 \%$ que actualmente se permite en el Reglamento del Distrito Federal. 
Cabe señalar que las aparentes discrepancias obtenidas entre los edificios de tres y de cinco niveles (figura 19) tienen justificación en el distinto impacto de las deformaciones por cortante entre ambos modelos; de hecho, ya se habían observado en el estudio de sistemas simétricos (Cano 2005). El impacto de las deformaciones por cortante de los muros del segundo nivel es mucho mayor en los edificios de tres niveles que en los de cinco niveles, dado que los muros del segundo nivel se comportan elásticamente en los edificios de tres niveles mientras que en los edificios de cinco niveles están agrietados (figura 8).

\section{Evaluación para el agrietamiento de los muros sólo en los niveles inferiores}

Considérese la condición cercana al estado último de diseño establecido en las NTCM-2004 y observado experimentalmente en muros de mampostería confinada (por ejemplo, Ruiz y Alcocer 1998, Arias et al. 2004), donde los edificios se encuentran parcialmente agrietados, es decir, que todos los muros del primer nivel se encuentran agrietados y todos los muros de los dos niveles restantes permanecen elásticos para los edificios de tres niveles y para los edificios de cinco niveles, todos los muros de los dos primeros niveles se encuentran agrietados y todos los muros de los tres niveles restantes se comportan elásticamente (fig 8). Tomando el $\mathrm{F}_{\mathrm{AEPA}}$ de la ecuación 19 y conforme a los resultados obtenidos tanto para los muros perimetrales críticos como para los muros centrales para todas las excentricidades en estudio (Tena y López 2006, López 2007), aunque en este trabajo sólo se presentan las gráficas para 10\% para los edificios de tres y cinco niveles (figura 20), se pueden hacer las siguientes observaciones generales.

Se observa a partir de la figura 20 que, para este desempeño estructural y factor de área efectiva propuesto, se obtienen mejores aproximaciones para los edificios de tres niveles (figura 20a) que para los modelos de cinco niveles (figura 20b). Se observa en general que la relación $\mathrm{V}_{3 \mathrm{D}} / \mathrm{V}_{\mathrm{MS}}$ tiende a estar cercana a 1.0 para las relaciones de aspecto $(H / L)$ consideradas, lo que sugiere que, para este caso, el método simplificado tiene una coincidencia razonable con un análisis riguroso para la mayoría de las relaciones de aspecto $(H / L)$ de los muros en estudio.

De la figura 20a, para los modelos de tres niveles y una $e_{s}=10 \%$, que es el límite fijado actualmente por las NTCM-2004, se aprecia que la máxima subestimación del método simplificado es de aproximadamente 1.3 veces en los muros perimetrales (MP) cuando los muros perimetrales y centrales tienen una relación de aspecto $H / L=0.5$, la cual es muy razonable. De la figura $20 \mathrm{~b}$ se aprecia que las peores aproximaciones se obtienen para los muros centrales (MC) de los edificios de cinco niveles cuando su relación de aspecto es $H / L=0.5$ y la relación de aspecto de los muros perimetrales está dentro del intervalo $0.9 \leq H / L \leq 2.2$. Cabe señalar que las diferencias en la forma de las curvas entre los modelos de tres $\mathrm{y}$ de cinco niveles se debe al diferente impacto de las deformaciones por cortante entre ambas modelaciones, dado de los muros del segundo nivel se modelaron como elásticos en los edificios de tres niveles, mientras que en los edificios de cinco niveles se consideraron agrietados (figura 8).

Desde un análisis que se enfoca más a definir el valor límite de la excentricidad estática que debiera aceptársele al método simplificado cuando se agrietan exclusivamente los muros de los niveles inferiores del edificio (figura 8), permitiendo que el método simplificado subestime hasta en un 30\% ó $40 \%$ la fuerza cortante $\left(\mathrm{V}_{3 \mathrm{D}} / \mathrm{V}_{\mathrm{MS}} \leq 1.4\right)$, se pueden hacer las siguientes observaciones cuando uno valora la propuesta de $F_{A E P A}($ ecuación 19).

Para los edificios de tres niveles, el método simplificado es aceptable para todas las relaciones de aspecto de los muros centrales cuando $\mathrm{e}_{\mathrm{s}} \leq 10 \%$ y aún para $\mathrm{e}_{\mathrm{s}}=20 \%$ (no mostrado). Sin embargo, los resultados entre los edificios de cinco y tres niveles varían considerablemente, siendo menos favorables las estimaciones de los modelos de cinco niveles, por lo que estos resultados también deben ponderarse para establecer limitaciones de uso al método simplificado. Conforme a lo anterior y para fines prácticos, 
Revisión y actualización del método simplificado de análisis de estructuras de mampostería de los reglamentos de ...

se propone el uso del método simplificado para realizar el análisis hasta para una excentricidad estática $e_{s}=10 \%$ (figura 20), el límite actualmente permitido, para todas las relaciones de aspecto de los muros centrales y perimetrales estudiadas, es decir, $0.5 \leq H / L \leq 2.5$.

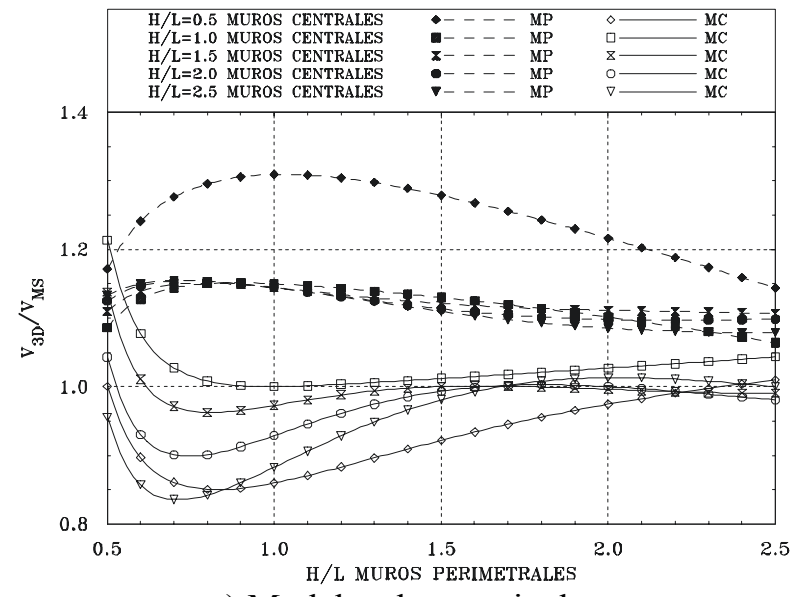

a) Modelos de tres niveles

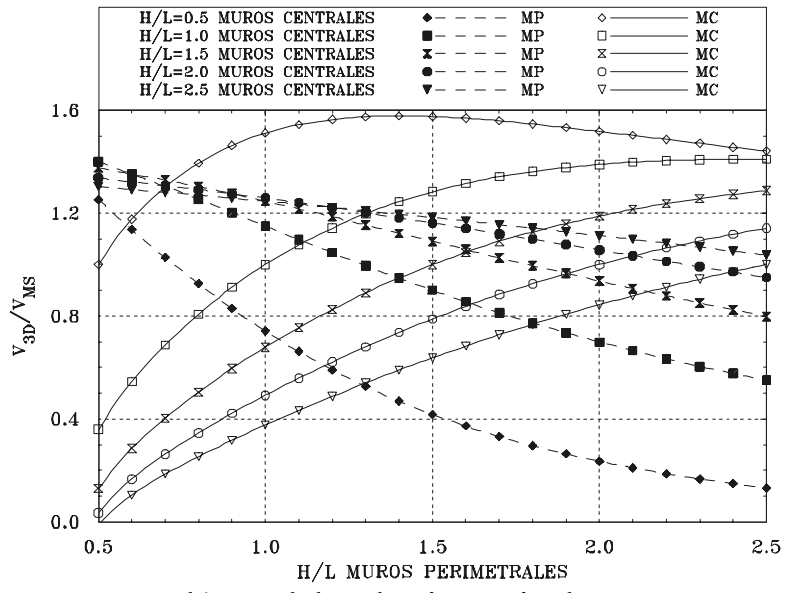

b) Modelos de cinco niveles

Figura 20. Relación de fuerzas cortantes $\mathrm{V}_{3 \mathrm{D}} / \mathrm{V}_{\mathrm{MS}}$ para los muros del primer nivel de los edificios de la figura 17 cuando $e_{s}=10 \%$, el comportamiento es parcialmente agrietado y se emplean el factor de área efectiva $\mathrm{F}_{\mathrm{AEPA}}$ propuesto (ec 19). Las curvas se trazan para las relaciones de aspecto $H / L$ de los muros centrales indicados en las leyendas.

\section{Evaluación para el agrietamiento de los muros en todos los niveles}

Considérese la condición más crítica que se ha observado en diversos sismos para edificios de mampostería no confinada ni reforzada, que es que los edificios se encuentran totalmente agrietados, es decir, que todos los muros se encuentran agrietados en todos los niveles (figura 9). Tomando el $F_{A E T A}$ propuesto (ecuación 20) y conforme a los resultados obtenidos tanto para los muros perimetrales críticos como los muros centrales para todas las excentricidades en estudio (López 2007), aunque en este trabajo sólo se presentan las gráficas para 10\% para los edificios de tres y cinco niveles (figura 21), se pueden hacer las siguientes observaciones.

Desde la óptica para definir el valor límite de la excentricidad estática que debiera permitírsele al método simplificado cuando se agrietan todos los muros, aceptando que el método simplificado subestime hasta en un $40 \%$ la fuerza cortante $\left(\mathrm{V}_{\mathrm{D}} / \mathrm{V}_{\mathrm{MS}} \leq 1.4\right)$, se pueden hacer las siguientes observaciones cuando uno valora la propuesta del $F_{A E T A}$ (ecuación 20) para los edificios de tres y cinco niveles. Para este caso, de acuerdo con los resultados del estudio paramétrico, el valor límite de la excentricidad estática $\left(e_{s}\right)$ propuesto por las NTCM-2004 vigentes $e_{s}=10 \%$ (figura 21) es razonable para todos los casos considerados. Al parecer, pudiera incluso aceptarse el uso del método simplificado para excentricidades estáticas mayores a 10\% cuando todos los muros estén totalmente agrietados de manera uniforme en planta y elevación (no mostrado, Tena y López 2006), pero se considera juicioso mantener el límite actualmente propuesto. Esto se debe a la reflexión de entender que, previo a este estado último de agrietamiento generalizado de los muros en planta y en elevación, los muros experimentarán estados elásticos y/o de agrietamiento parcial con una distribución no necesariamente uniforme en planta y/o elevación, por lo que las estimaciones finales de fuerzas pudieran diferir substancialmente con las 
estimadas en este estudio utilizando análisis estáticos equivalentes, que no puede representar adecuadamente un fenómeno dinámicamente tan complejo.

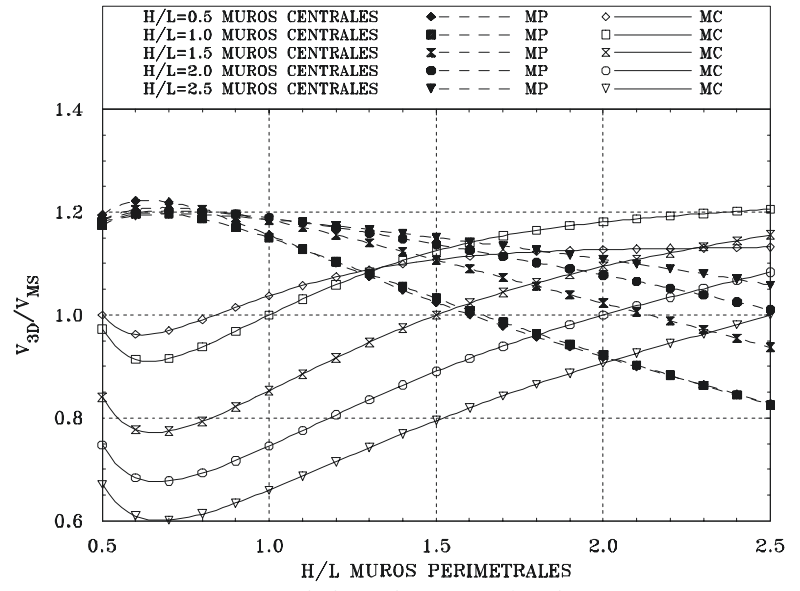

a) Modelos de tres niveles

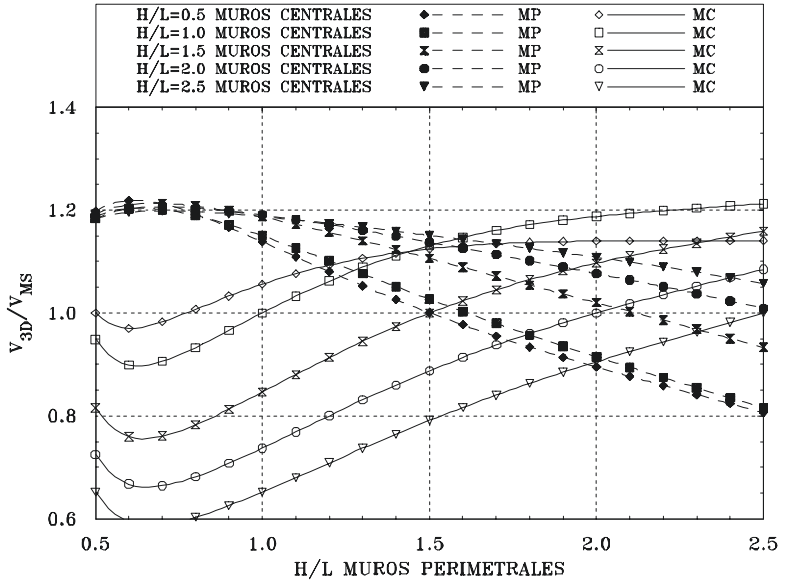

b) Modelos de cinco niveles

Figura 21. Relación de fuerzas cortantes $\mathrm{V}_{3 \mathrm{D}} / \mathrm{V}_{\mathrm{MS}}$ para los muros del primer nivel de los edifícios de la figura 17 cuando $e_{s}=10 \%$, el comportamiento es totalmente agrietado y se emplean el factor de área efectiva $\mathrm{F}_{\mathrm{AETA}}$ propuesto (ec 20). Las curvas se trazan para las relaciones de aspecto $H / L$ de los muros centrales indicados en las leyendas.

\section{EVALUACIÓN DE LOS FACTORES DE ÁREA EFECTIVA Y DE LOS VALORES LÍMITE DE EXCENTRICIDAD ESTÁTICA RECOMENDADOS}

Con el fin de valorar la potencial diferencia en el diseño final para resistir cortantes por sismo de estructuras de mampostería confinada cuando se aplica el método simplificado con respecto a un análisis riguroso, se consideró un edificio típico de mampostería cuya planta tipo se muestra en la figura 22.

El edificio en cuestión está destinado a oficinas y cuenta con cinco niveles con altura típica de entrepiso $h=2.50 \mathrm{~m}$. Los muros son de ladrillo con $f^{*}=100 \mathrm{~kg} / \mathrm{cm}^{2}$ y espesor $t=12.5 \mathrm{~cm}$, unidos con mortero tipo I, por lo que de acuerdo con las NTCM-2004 $f^{*}{ }_{m}=40 \mathrm{~kg} / \mathrm{cm}^{2}$ y $v^{*}{ }_{m}=3.5 \mathrm{~kg} / \mathrm{cm}^{2}$. El módulo de elasticidad E y G se obtiene conforme a las expresiones propuestas por las NTCM-2004 (implícitamente se considera $v=0.25$ ). Los castillos son de $12.5 \times 12.5 \mathrm{~cm}$ con refuerzo mínimo según las NTCM-2004. El coeficiente sísmico reducido para edificios de $12.5 \mathrm{~m}$ hechos con muros de mampostería de piezas macizas para la zona III es $c=0.19$ según la Tabla 7.1 de las NTCS-2004. El espesor de la losa de concreto es de $10 \mathrm{~cm}$, y está perimetralmente apoyada sobre dalas de cerramiento de $15 \times 25 \mathrm{~cm}$ indicadas con líneas punteadas en la figura 22 .

Con base en lo anterior, se calcularon las fuerzas laterales actuantes (aplicadas en dirección X, que es normal a la dirección Y, donde existen excentricidades), las excentricidades estáticas asociadas y las fuerzas cortantes que absorben los muros en dirección $\mathrm{X}$, y con base en ello se realizaron prediseños, como se discute en las siguientes secciones. Cabe señalar que para hacer una comparación en igualdad de circunstancias con el método simplificado, en ambos métodos de análisis se consideró exclusivamente la acción de las fuerzas laterales en una sola dirección. Por lo tanto, no se consideraron en los análisis estáticos rigurosos las combinaciones de carga asociadas a los efectos bidireccionales que le demandarían las NTCS-2004. 


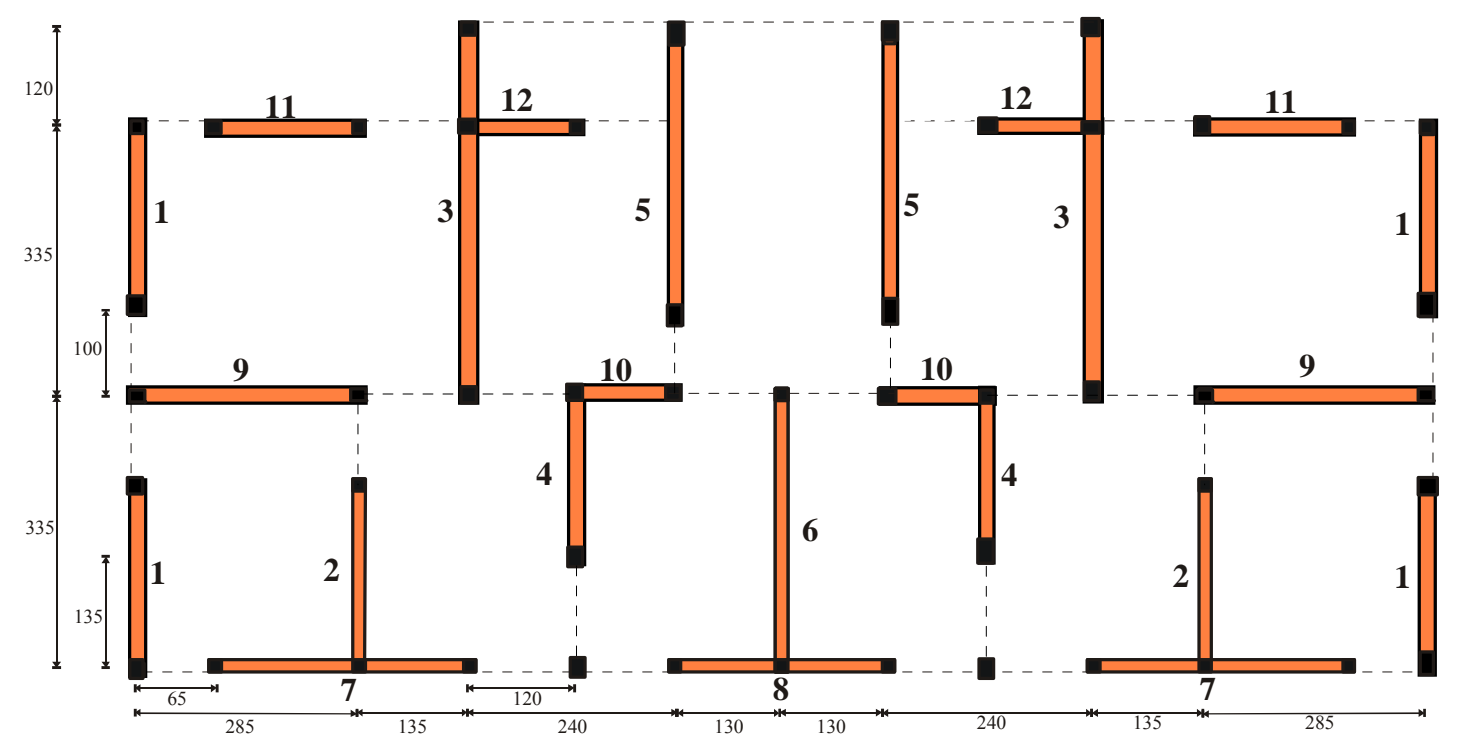

Figura 22. Planta tipo del edificio en estudio

\section{Comparación de las excentricidades estáticas}

Las excentricidades estáticas en dirección Y se reportan en la tabla 1, donde MS identifica al método simplificado y "Damy" a un análisis estático tridimensional riguroso donde se aplica el método matricial propuesto por Damy para valorar las excentricidades estáticas. Cuando las excentricidades estáticas son calculadas usando el método simplificado de análisis, se observa que: (1) ninguna de ellas es menor del $10 \%$ con respecto a la planta que es paralela a la dirección donde se sitúa dicha excentricidad, (2) las menores excentricidades calculadas con el método simplificado se obtienen cuando se emplea el $F_{A E P A}$ (parcialmente agrietado), mientras que las mayores excentricidades se presentan cuando éstas son calculadas usando el $F_{A E}$ (totalmente elástico), (3) en ninguno de los tres casos considerados coinciden las excentricidades calculadas entre el método simplificado y el método riguroso, (4) la estimación de la excentricidad con el método simplificado es conservadora para todos los niveles sólo para el caso del $F_{A E}$ para comportamiento totalmente elástico propuesto y es no conservadora para los $F_{A E P A}$ y $F_{A E T A}$ propuestos.

Tomando en cuenta que los factores de área efectiva propuestos toman como base estimar razonablemente las fuerzas cortantes de los muros de planta baja (nivel 1), es en este nivel donde, con rigor, debe valorarse el valor predicho para la excentricidad estática $e_{s}$. En este sentido, las estimaciones más cercanas corresponden al $F_{A E P A} \mathrm{y}$ al $F_{A E T A}$.

\section{Comparación de prediseños de los muros}

Debe recordarse que, para fines de este estudio, lo realmente importante para decidir si se puede o no aplicar el método simplificado es la estimación de las excentricidades estáticas de manera aproximada con la fórmula propuesta para este método, es decir, con los factores de área efectiva propuestos. 
Arturo Tena Colunga, Jesús Cano Licona, Arturo López Blancas y Miguel Ángel Pérez Osornio

Tabla 1. Excentricidades estáticas $\left(e_{s i}\right)$ en \% en la dirección corta $(\mathrm{Y})$ del edificio en estudio

\begin{tabular}{ccccccc}
\hline \multirow{2}{*}{ Nivel } & \multicolumn{2}{c}{$\boldsymbol{F}_{\boldsymbol{A E E}}$ Tot. Elástico } & \multicolumn{2}{c}{$\boldsymbol{F}_{\boldsymbol{A E P A} \text { A }}$ Agr. Parcial } & \multicolumn{2}{c}{$\boldsymbol{F}_{\boldsymbol{A E T A}}$ Agr. Total } \\
\cline { 2 - 7 } & MS & Damy & MS & Damy & MS & Damy \\
\hline $\mathbf{5}$ & 20 & 16.51 & 10.8 & 28.78 & 11.15 & 22.82 \\
$\mathbf{4}$ & 20 & 10.36 & 10.8 & 25.08 & 11.15 & 19.76 \\
$\mathbf{3}$ & 20 & 4.26 & 10.8 & 16.73 & 11.15 & 17.57 \\
$\mathbf{2}$ & 20 & 3.08 & 10.8 & 0.24 & 11.15 & 19.39 \\
$\mathbf{1}$ & 20 & 2.25 & 10.8 & 11.03 & 11.15 & 13.99 \\
\hline
\end{tabular}

Por lo tanto, se estimaron las fuerzas cortantes que deben resistir los muros de planta baja con las distintas variantes del método simplificado con las obtenidas con análisis estáticos tridimensionales rigurosos. A partir de ello, se compararon estas fuerzas demandadas con respecto a su resistencia de diseño $\left(V_{m R}\right)$ conforme a las NTCM-2004:

$$
V_{m R}=F_{R}\left(0.5 v_{m}^{*} A_{T}+0.3 P\right) \leq 1.5 F_{R} v_{m}^{*} A_{T}
$$

donde $v^{*}{ }_{m}=3.5 \mathrm{~kg} / \mathrm{cm}^{2}$ es la resistencia a compresión diagonal de la mampostería para diseño, $P$ es la carga vertical de compresión que actúa sobre el muro, $A_{T}$ es el área bruta de la sección transversal del muro, que debe incluir los castillos en el caso de la mampostería confinada, pero sin transformar el área transversal y $F_{R}$ es el factor de reducción por resistencia que vale 0.7 para mampostería confinada y 0.4 para mampostería no confinada ni reforzada.

En la mayor parte de los casos en estudio, los muros de mampostería no eran capaces de resistir la fuerza cortante demandada dependiendo exclusivamente de la mampostería, por lo que fue necesario proveer de refuerzo horizontal. Se determinó el acero de refuerzo horizontal requerido para resistir el déficit de cortante conforme lo establecen las NTCM-2004, observando los porcentajes de refuerzo mínimo y máximo, además de requisitos de detallado y de uniformidad. Esto se hizo de la siguiente manera. A partir de la fuerza cortante última que deben resistir los muros de mampostería, $V_{u}$, se determina la fuerza cortante que debe tomar el refuerzo horizontal, $V_{s R}$ :

$$
V_{u}=V_{m R}+V_{s R} \rightarrow \quad V_{s R}=V_{u}-V_{m R}
$$

A partir de $V_{s R}$ se calcula la cuantía de acero de refuerzo horizontal $p_{h}$ :

$$
V_{s R}=F_{R} \eta p_{h} f_{y h} A_{T} \rightarrow \quad p_{h}=\frac{V_{s R}}{F_{R} \eta f_{y h} A_{T}}
$$

donde $F_{R}$ y $A_{T}$ ya se definieron con anterioridad, $f_{y h}$ es el esfuerzo de fluencia especificado de diseño del acero de refuerzo horizontal y $\eta$ es un factor de eficiencia del refuerzo horizontal según las NTCM-2004 que varía entre 0.6 para cuantías de refuerzo bajas y 0.2 para cuantías de refuerzo altas. La cuantía de acero de refuerzo horizontal, $p_{h}$, debe cumplir con los siguientes requisitos mínimos: 
Revisión y actualización del método simplificado de análisis de estructuras de mampostería de los reglamentos de ...

$$
p_{h} \geq\left\{\begin{array}{l}
\frac{3}{f_{y h}} \\
\frac{V_{m R}}{F_{R} f_{y h} A_{T}}
\end{array}\right.
$$

En ningún caso $p_{h}$ sobrepasará los siguientes máximos para muros con piezas macizas:

$$
p_{h} \leq\left\{\begin{array}{l}
0.3 \frac{f_{m} *}{f_{y h}} \\
\frac{12}{f_{y h}}
\end{array}\right.
$$

En las ecuaciones 23 y 24, las unidades de $f_{y h}$ son $\mathrm{kg} / \mathrm{cm}^{2}$. Por requisitos de detallado, la separación máxima del refuerzo horizontal $s_{h}$ no debe ser mayor de cuatro hiladas ni de $60 \mathrm{~cm}$. En el caso de los tabiques aquí considerados, cuyo espesor típico es de $6.5 \mathrm{~cm}$, y la junta típica de mortero tiene un espesor de $1 \mathrm{~cm}$, rigen las cuatro hiladas como separación máxima.

En las tablas 2 a 5 se resume el prediseño de las áreas de acero requeridas para satisfacer las demandas de fuerza cortante para la zona III de las NTCS-2004. En dichas tablas, $V_{m R d x}$ identifica a la resistencia a cortante de diseño de la mampostería, que se calculó conforme a la ecuación 21 y donde la carga axial de compresión de diseño corresponde a la carga muerta más viva instantánea que soporta cada muro conforme a una bajada de cargas por áreas tributarias, como se reporta en López (2007), $V_{u x}$ es la fuerza cortante actuante (demandada por el análisis) y $V_{S R}$ es la fuerza cortante que, en su caso, debe tomar el refuerzo horizontal. En la columna "SRH", se identifica si el muro en cuestión resiste la fuerza cortante sin refuerzo horizontal: si se escribe "No" significa que el muro no resiste y necesita acero de refuerzo, si se escribe "Si", quiere decir que el muro no requiere refuerzo; sin embargo, por uniformidad, se decidió proporcionarle refuerzo, bajo la premisa de que "o todos los muros se refuerzan, o ninguno", práctica recomendada para evitar errores constructivos debidos a una supervisión inadecuada o mala interpretación de planos estructurales. Por otro lado, Acero (") indica la cantidad de acero de diseño, tomando en cuenta que se satisfacen todos los requisitos de diseño en cuanto a cuantías máximas y mínimas, separaciones, detallado y ajuste a calibres comerciales (dados en fracciones de pulgada), y "s (hiladas)" es la separación del acero de refuerzo en hiladas, atendiendo requisitos mínimos y máximos por cuestiones de detallado. En todos los casos se consideró que el refuerzo horizontal es con base en alambres corrugados laminados en frío con $f_{y}=6,000 \mathrm{~kg} / \mathrm{cm}^{2}$, empleando para ello calibres comerciales. Los alambres se colocan típicamente en pares en juntas, anclados al acero de refuerzo vertical de los castillos, como se establece en los detalles constructivos que se ilustran en las NTCM-2004.

Cabe señalar que los prediseños presentados son de carácter ilustrativo atendiendo requisitos reglamentarios, pero no constituyen diseños finales. Para un diseño final que se edifique se deberían ajustar otras variables constructivas de índole práctico, por ejemplo, decidir si se uniformiza la separación con que se colocará el refuerzo horizontal y el calibre de los alambres corrugados laminados en frío, por ejemplo. Además, en el caso de los análisis con el método estático, conforme a las NTCS-2004 se deberían considerar las combinaciones de carga por sismo considerando efectos bidireccionales y la 
alternancia de signo, lo cual seguramente llevará a un diseño más conservador de todos los muros. Como ya se mencionó, esto no se efectuó para comparar las aproximaciones del método simplificado en igualdad de circunstancias.

En la tabla 2 se presentan los prediseños de los muros de PB para comportamiento totalmente elástico. Para este comportamiento, se aprecia que las cuantías de refuerzo demandadas y los armados ajustados de un prediseño basado en análisis con el método simplificado y otro basado en análisis con métodos rigurosos fueron distintos entre sí. En otras palabras, la disparidad importante que existe entre ambos métodos es evidente en la cuantía de refuerzo demandada, no sólo en las fuerzas cortantes $\left(V_{u x i}\right)$ que actúan en cada muro. Esto es una consecuencia lógica de la gran diferencia existente entre las excentricidades estáticas calculadas conforme al método simplificado y los $F_{A E}$ propuestos para comportamiento totalmente elástico con respecto al método riguroso (tabla 1). Además, las excentricidades estáticas calculadas conforme al método simplificado (tabla 1) se encuentren muy por encima de los valores límite propuestos para obtener una aproximación razonable $\left(e_{s}<0.05\right)$. Por ello, los diseños en estos casos difieren notablemente, ya que la no uniformidad de las cuantías de refuerzo demandadas en los distintos muros en un análisis riguroso incluyen a las fuerzas cortantes adicionales por los efectos de la torsión asociadas a esta excentricidad, lo cual no se considera en el método simplificado. En este caso, lo razonable sería diseñar conforme al método riguroso y no hacer diseños conforme al método simplificado.

Tabla 2. Prediseño de los muros de PB en la dirección X (figura 22), utilizando el $F_{A E}$ propuesto para el método simplificado para comportamiento totalmente elástico

\begin{tabular}{|c|c|c|c|c|c|c|c|c|c|c|c|c|}
\hline \multirow{2}{*}{$\begin{array}{r}\text { Muro } \\
\text { Tipo }\end{array}$} & \multicolumn{6}{|c|}{ Método Simplificado $\left(F_{A E}\right.$ propuesto $)$} & \multicolumn{6}{|c|}{ Análisis Estático Tridimensional (Damy) } \\
\hline & $\begin{array}{l}V_{m R d x} \\
\text { (Ton) }\end{array}$ & $\begin{array}{c}V_{u x} \\
\text { (Ton) }\end{array}$ & SRH & $\begin{array}{c}V_{S R} \\
\text { (Ton) }\end{array}$ & $\begin{array}{c}\rho_{h} \\
\text { diseño }\end{array}$ & $\begin{array}{c}\text { Acero (") y s } \\
\text { (hiladas) }\end{array}$ & $\begin{array}{l}V_{m R d x} \\
\text { (Ton) }\end{array}$ & $\begin{array}{c}V_{u x} \\
\text { (Ton) }\end{array}$ & SRH & $\begin{array}{c}V_{S R} \\
\text { (Ton) }\end{array}$ & $\begin{array}{c}\rho_{h} \\
\text { diseño }\end{array}$ & $\begin{array}{c}\text { Acero (") y s } \\
\text { (hiladas) }\end{array}$ \\
\hline 7 & 9.89 & 19.12 & No & 9.23 & 0.0008 & 2 al 3/16@4 & 9.89 & 10.89 & No & 1.00 & $0.0005^{1}$ & 2 al5/32@4 \\
\hline 8 & 3.56 & 11.02 & No & 7.46 & 0.0009 & 2 al 3/16@4 & 3.56 & 10.40 & No & 6.85 & 0.0008 & 2 al3/16@4 \\
\hline 9 & 7.29 & 13.34 & No & 6.05 & 0.0006 & 2 al 5/32@4 & 7.29 & 9.58 & No & 2.28 & $0.0005^{1}$ & 2 al 5/32@4 \\
\hline 10 & 1.46 & 2.03 & No & 0.57 & $0.0005^{1}$ & 2 al 5/32@4 & 1.46 & 8.28 & No & 6.82 & 0.0018 & 2 al3/16@2 \\
\hline 11 & 6.28 & 7.60 & No & 1.32 & $0.0005^{1}$ & 2 al 5/32@4 & 6.28 & 8.25 & No & 1.98 & $0.0005^{1}$ & 2 al 5/32@4 \\
\hline 12 & 3.13 & 2.03 & $\mathrm{Si}$ & -- & $0.0005^{1}$ & 2 al 5/32@4 & 3.13 & 7.42 & No & 4.29 & 0.0011 & 2 al 5/32@2 \\
\hline
\end{tabular}

Cuando se considera el caso más representativo para mampostería confinada conforme a la práctica mexicana, el parcialmente agrietado (tablas 3 y 4), se observa que la diferencia en las cuantías de refuerzo demandadas y los armados ajustados es menor con respecto al caso anterior ( $F_{A E}$ totalmente elástico) y también se cumple que los armados para resistir las fuerzas laterales en los muros de mampostería en el segundo nivel (tabla 3) son más parecidos que los del primer nivel (tabla 4). Estos resultados confirman que cuando la excentricidad estática calculada conforme al método simplificado y el $F_{A E P A}$ propuesto (tabla 1) se encuentra cercana al valor límite propuesto $\left(e_{s} \leq 0.10\right)$, los prediseños hechos con el método simplificado deben resultar razonables cuando se comparan con diseños basados en análisis rigurosos en igualdad de circunstancias (mismas fuerzas laterales de diseño), como lo son para este edificio en particular, que incluye a 12 tipos de muros diferentes, es decir, 12 distintas relaciones de aspecto $H / L$ de los muros.

Por último, en la tabla 5 se presentan los resultados para el nivel de desempeño de muros totalmente agrietados, más representativo del comportamiento observado en sismos intensos en estructuras de mampostería no reforzada y, por tanto, resulta interesante valorarlo para la modalidad de mampostería no confinada ni reforzada que establecen las NTCM-2004. 
Revisión y actualización del método simplificado de análisis de estructuras de mampostería de los reglamentos de ...

Tabla 3. Prediseño de los muros del nivel 2 en la dirección X (figura 22), utilizando el $F_{A E P A}$ propuesto para el método simplificado para comportamiento parcialmente agrietado

\begin{tabular}{|c|c|c|c|c|c|c|c|c|c|c|c|c|}
\hline \multirow{2}{*}{$\begin{array}{r}\text { Muro } \\
\text { Tipo }\end{array}$} & \multicolumn{6}{|c|}{ Método Simplificado $\left(F_{A E P A}\right.$ propuesto) } & \multicolumn{6}{|c|}{ Análisis Estático Tridimensional (Damy) } \\
\hline & $\begin{array}{l}V_{m R d x} \\
\text { (Ton) }\end{array}$ & $\begin{array}{c}V_{u x} \\
\text { (Ton) }\end{array}$ & SRH & $\begin{array}{c}V_{S R} \\
\text { (Ton) }\end{array}$ & $\begin{array}{c}\rho_{h} \\
\text { diseño }\end{array}$ & $\begin{array}{c}\begin{array}{c}\text { Acero (") y s } \\
\text { (hiladas) }\end{array} \\
\text { (a) }\end{array}$ & $\begin{array}{l}V_{m R d x} \\
\text { (Ton) }\end{array}$ & $\begin{array}{c}V_{u x} \\
\text { (Ton) }\end{array}$ & SRH & $\begin{array}{c}V_{S R} \\
\text { (Ton) }\end{array}$ & $\begin{array}{c}\rho_{h} \\
\text { diseño }\end{array}$ & $\begin{array}{c}\text { Acero (") y s } \\
\text { (hiladas) }\end{array}$ \\
\hline 7 & 8.79 & 12.31 & No & 3.52 & $0.0005^{1}$ & 2 al 5/32@4 & 8.79 & 12.47 & No & 3.68 & $0.0005^{1}$ & 2 al 5/32@4 \\
\hline 8 & 3.25 & 9.69 & No & 6.44 & 0.0008 & 2 al 3/16@4 & 3.25 & 9.38 & No & 6.13 & 0.0007 & 2 al 3/16@4 \\
\hline 9 & 6.52 & 10.40 & No & 3.88 & $0.0005^{1}$ & 2 al 5/32@4 & 6.52 & 11.12 & No & 4.60 & 0.0005 & 2 al 5/32@4 \\
\hline 10 & 1.37 & 5.00 & No & 3.63 & 0.0009 & $2 \mathrm{al} 3 / 16 @ 4$ & 1.37 & 4.08 & No & 2.71 & 0.0007 & 2 al 3/16@4 \\
\hline 11 & 5.77 & 8.49 & No & 2.72 & $0.0005^{1}$ & $2 \mathrm{al} 5 / 32 @ 4$ & 5.77 & 9.26 & No & 3.49 & 0.0005 & 2 al 5/32@4 \\
\hline 12 & 2.88 & 5.00 & No & 2.12 & 0.0005 & 2 al 5/32@4 & 2.88 & 4.43 & No & 1.55 & $0.0005^{1}$ & 2 al 5/32@ 4 \\
\hline
\end{tabular}

Tabla 4. Prediseño de los muros de PB en la dirección X (figura 22), utilizando el $F_{A E P A}$ propuesto para el método simplificado para comportamiento parcialmente agrietado

\begin{tabular}{|c|c|c|c|c|c|c|c|c|c|c|c|c|}
\hline \multirow{2}{*}{$\begin{array}{r}\text { Muro } \\
\text { Tipo }\end{array}$} & \multicolumn{6}{|c|}{ Método Simplificado ( $F_{A E P A}$ propuesto) } & \multicolumn{6}{|c|}{ Análisis Estático Tridimensional (Damy) } \\
\hline & $\begin{array}{l}V_{m R d x} \\
\text { (Ton) } \\
\end{array}$ & $\begin{array}{c}V_{u x} \\
\text { (Ton) }\end{array}$ & SRH & $\begin{array}{c}V_{S R} \\
\text { (Ton) }\end{array}$ & $\begin{array}{c}\rho_{h} \\
\text { diseño }\end{array}$ & $\begin{array}{c}\text { Acero (") y s } \\
\text { (hiladas) }\end{array}$ & $\begin{array}{l}V_{m R d x} \\
\text { (Ton) }\end{array}$ & $\begin{array}{c}V_{u x} \\
\text { (Ton) }\end{array}$ & SRH & $\begin{array}{c}V_{S R} \\
\text { (Ton) }\end{array}$ & $\begin{array}{c}\rho_{h} \\
\text { diseño }\end{array}$ & $\begin{array}{c}\text { Acero (") y s } \\
\text { (hiladas) }\end{array}$ \\
\hline 7 & 9.89 & 13.27 & No & 3.38 & $0.0005^{1}$ & 2 al 5/32@4 & 9.89 & 18.09 & No & 8.20 & 0.0007 & 2 al 3/16@4 \\
\hline 8 & 3.56 & 10.44 & No & 6.88 & 0.0008 & 2 al 3/16@4 & 3.56 & 9.56 & No & 6.00 & 0.0007 & 2 al 3/16@4 \\
\hline 9 & 7.29 & 11.21 & No & 3.92 & $0.0005^{1}$ & 2 al 5/32@4 & 7.29 & 13.11 & No & 5.82 & 0.0006 & 2 al 5/32@4 \\
\hline 10 & 1.46 & 5.39 & No & 3.93 & 0.0009 & 2 al 3/16@4 & 1.46 & 2.25 & No & 0.79 & $0.0005^{1}$ & 2 al 5/32@4 \\
\hline 11 & 6.28 & 9.15 & No & 2.87 & $0.0005^{1}$ & 2 al 5/32@4 & 6.28 & 8.82 & No & 2.55 & $0.0005^{1}$ & 2 al 5/32@4 \\
\hline 12 & 3.13 & 5.39 & No & 2.26 & 0.0005 & 2 al 5/32@4 & 3.13 & 2.57 & $\mathrm{Si}$ & -- & $0.0005^{1}$ & 2 al 5/32@4 \\
\hline
\end{tabular}

Tabla 5. Prediseño de los muros de PB en la dirección X (figura 28), utilizando el $F_{A E T A}$ propuesto para el método simplificado para comportamiento totalmente agrietado

\begin{tabular}{|c|c|c|c|c|c|c|c|c|c|c|c|c|}
\hline \multirow{2}{*}{$\begin{array}{r}\text { Muro } \\
\text { Tipo }\end{array}$} & \multicolumn{6}{|c|}{ Método Simplificado $\left(F_{A E T A}\right.$ propuesto) } & \multicolumn{6}{|c|}{ Análisis Estático Tridimensional (Damy) } \\
\hline & $\begin{array}{l}V_{m R d x} \\
\text { (Ton) }\end{array}$ & $\begin{array}{c}V_{u x} \\
\text { (Ton) }\end{array}$ & SRH & $\begin{array}{c}V_{S R} \\
\text { (Ton) }\end{array}$ & $\begin{array}{c}\rho_{h} \\
\text { diseño }\end{array}$ & Observación & $\begin{array}{l}V_{m R d x} \\
\text { (Ton) }\end{array}$ & $\begin{array}{c}V_{u x} \\
\text { (Ton) }\end{array}$ & SRH & $\begin{array}{c}V_{S R} \\
\text { (Ton) }\end{array}$ & $\begin{array}{c}\rho_{h} \\
\text { diseño }\end{array}$ & Observación \\
\hline 7 & 5.65 & 26.94 & No & 21.29 & $0.0033^{2}$ & No pasa & 5.65 & 29.20 & No & 23.55 & $0.0037^{2}$ & No pasa \\
\hline 8 & 2.03 & 21.04 & No & 19.01 & $0.0041^{2}$ & No pasa & 2.03 & 20.08 & No & 18.05 & $0.0039^{2}$ & No pasa \\
\hline 9 & 4.17 & 22.66 & No & 18.49 & $0.0036^{2}$ & No pasa & 4.17 & 24.76 & No & 20.59 & $0.0040^{2}$ & No pasa \\
\hline 10 & 0.83 & 10.42 & No & 9.59 & $0.0044^{2}$ & No pasa & 0.83 & 7.58 & No & 6.75 & $0.0031^{2}$ & No pasa \\
\hline 11 & 3.59 & 18.30 & No & 14.71 & $0.0037^{2}$ & No pasa & 3.59 & 19.42 & No & 15.83 & $0.0040^{2}$ & No pasa \\
\hline 12 & 1.79 & 10.42 & No & 8.63 & $0.0040^{2}$ & No pasa & 1.79 & 8.26 & No & 6.47 & $0.0030^{2}$ & No pasa \\
\hline
\end{tabular}

Cabe señalar lo siguiente para la mampostería no confinada ni reforzada. Conforme a las NTCM2004, no se debe emplear el método simplificado para esta modalidad en estructuras de más de tres niveles, pero se hace caso omiso sólo en esta ocasión con fines exclusivamente ilustrativos. De acuerdo con las NTCM-2004, la mampostería no confinada ni reforzada se debe diseñar considerando un comportamiento elástico, por lo cual $Q=1$ y esto implica que el coeficiente sísmico reducido que debe emplearse en el método simplificado para la zona III de las NTCS-2004 es $c=0.38$, lo cual explica el por qué las fuerzas cortantes demandadas $\left(V_{u x}\right)$ son notablemente más grandes en esta tabla 5 con respecto a las anteriores. En este caso, el factor de reducción de resistencia a cortante es $F_{R}=0.4$, y esto explica, entre otras cosas, por qué la fuerza cortante resistida por los muros, $V_{m R d x}$, es menor que en los casos anteriores. Como consecuencia de ambos factores, la fuerza cortante que debe resistir el acero de refuerzo $\left(V_{S R}\right)$ y la cuantía de acero demandada $\left(\rho_{h}\right)$ se incrementan notablemente; de hecho supera notablemente a la cuantía de refuerzo máxima $\rho_{\max }=0.0020$ calculada conforme a las NTCM-2004 (ecuación 24). Por lo tanto, este edificio no podría diseñarse de ninguna manera como mampostería no confinada ni reforzada en la zona III de las NTCS-2004, lo cual es muy importante destacar, ya que ilustra cómo las disposiciones de las NTCM-2004 no permiten que se puedan diseñar y construir fácilmente estructuras altamente vulnerables en zonas de alto peligro sísmico. 
Si nos limitamos a comparar exclusivamente los resultados de los dos métodos de análisis (simplificado vs riguroso), como para los desempeños estructurales anteriormente considerados, se observa de la tabla 5 que las cuantías de refuerzo que son en teoría demandadas son similares entre si. Si observamos los cortantes actuantes para ambos métodos $\left(V_{u x}\right)$, que en este caso es quizá la información más relevante, dado que este comportamiento es representativo de mampostería sin refuerzo, la diferencia es pequeña. Estos resultados confirman, al igual que para el caso anterior, que cuando la excentricidad estática calculada conforme al método simplificado y el $F_{A E T A}$ propuesto (tabla 1 ) se encuentra cercano al límite propuesto $\left(e_{s} \leq 0.10\right)$, los diseños hechos conforme al método simplificado deben ser parecidos a los realizados conforme a un análisis riguroso, como ocurre para este edificio en particular.

\section{ACTUALIZACIONES DEL MÉTODO SIMPLIFICADO DEL MOC-2008}

Con base en los resultados de los estudios paramétricos a que se han hecho referencia y que se han presentado brevemente en este artículo, el nuevo Manual de Obras Civiles de Comisión Federal de Electricidad (MOC-2008 2009) incorpora en el método simplificado nuevos factores de área efectiva con respecto a su versión anterior (MOC-93 1993). El MOC-2008 estable a las ecuaciones 17 y 18, que se obtuvieron para una condición de comportamiento totalmente elástico (figura 7), para la revisión de estado límite de servicio y la ecuación 19 para un estado límite de prevención de colapso, correspondiente al agrietamiento de todos los muros en los niveles inferiores (figura 8).

Por simplicidad y congruencia, para edificios con estructuraciones convencionales, el MOC-2008 limita el valor de la excentricidad estática $e_{s}$ al $10 \%$ de la dimensión en planta en el sentido de que se mide la excentricidad, correspondiente al estado límite de prevención de colapso, dado que para la gran mayoría de este tipo de estructuras, éste último estado límite normalmente regirá el diseño por sismo.

Para estructuras con aislamiento sísmico, donde se establece que el comportamiento de la estructura por encima del sistema de aislamiento debe ser esencialmente elástica, el MOC-2008 establece que sólo deben utilizarse los factores de área efectiva para comportamiento elástico (ecuaciones 17 y 18) y limita el valor de la excentricidad estática $e_{s}$ al $5 \%$ de la dimensión en planta en el sentido de que se mide la excentricidad.

\section{COMENTARIOS FINALES Y CONCLUSIONES}

Se presentaron algunos de los aspectos más relevantes de una serie de investigaciones realizadas para revisar a fondo las disposiciones del método simplificado de análisis y diseño de los reglamentos de diseño sísmico de México.

Las investigaciones iniciales estudiaron el impacto que en la distribución de las fuerzas cortantes absorbidas por los muros tienen las deformaciones por cortante, por lo que se compararon las fuerzas cortantes que toman muros de mampostería con el método simplificado original con respecto a las obtenidas empleando un método riguroso de análisis estático tridimensional, tanto para sistemas simétricos como asimétricos. El estudio demostró que pueden existir diferencias importantes entre las fuerzas cortantes de entrepiso estimadas con el método simplificado original cuando se comparan con respecto a métodos rigurosos de análisis estructural tridimensional cuando los muros tienen distintas relaciones de aspecto $(H / L)$, aun para sistemas simétricos, por lo que se debían proponer factores de área efectiva $\left(F_{A E}\right)$ más realistas a cómo impactan las deformaciones por cortante en el análisis en todo el intervalo de relaciones de aspecto de los muros $(H / L)$, con base en estudios paramétricos. 
Revisión y actualización del método simplificado de análisis de estructuras de mampostería de los reglamentos de ...

Con base en los resultados de esos estudios paramétricos es que se proponen nuevos factores de área efectiva para tres niveles de desempeño estructural: (a) comportamiento totalmente elástico de los muros en planta y elevación $\left(F_{A E}\right)$, correspondiente a un estado límite de servicio, (b) comportamiento totalmente agrietado de los muros en los niveles inferiores, pero totalmente elástico en los niveles superiores $\left(F_{A E P A}\right)$, correspondiente a un estado límite de prevención de colapso para mampostería confinada y, (c) comportamiento totalmente agrietado de los muros en planta y elevación $\left(F_{A E T A}\right)$, correspondiente a un estado límite de prevención de colapso para mampostería no confinada ni reforzada. Como se demuestra en estas investigaciones y se ejemplifica en este estudio, las estimaciones de las fuerzas cortantes que toman los muros de mampostería con el método simplificado utilizando los factores de área efectiva propuestos mejoran notablemente las obtenidas con la propuesta de las NTCM-2004, cuando se comparan ambas con respecto a las obtenidas empleando un método riguroso de análisis estático tridimensional para todas las relaciones de aspecto $H / L$, y principalmente para el entrepiso crítico para diseño, que corresponde al nivel de planta baja.

Se realizó otro estudio paramétrico enfocado a proponer valores límite para la excentricidad estática en planta $\left(e_{s}\right)$ para la aplicación del método simplificado de análisis para estructuras con base en muros de mampostería para los distintos niveles de desempeño estructural indicados anteriormente, tomando como fundamento que las subestimaciones de las fuerzas cortantes obtenidas con el método simplificado para los muros críticos se encuentren dentro de límites aceptables, que permitan garantizar diseños razonablemente seguros de estructuras de mampostería. Por lo tanto, se propuso aceptar que con el método simplificado se obtenga una subestimación máxima de la fuerza cortante del $30 \%-40 \%$, que toma en cuenta tres aspectos esencialmente: (a) los muy bajos valores de $v^{*}{ }_{m}$ de las mamposterías mexicanas, donde una diferencia de esta magnitud puede redundar en que se requieran utilizar piezas o morteros de pegas distintos o, inclusive, se requiera de refuerzo horizontal, (b) cuando se emplea el método simplificado de análisis, no se toman en cuenta los efectos bidireccionales más que de una manera aproximada en el coeficiente sísmico de diseño propuesto para usar este método y, (c) cuando se usa el método simplificado no se amplifican las fuerzas adicionales por torsión para tomar en cuenta excentricidades accidentales y efectos dinámicos, como sí exigen las NTCS-2004 y otros reglamentos de diseño sísmico de México cuando se emplean otros métodos de análisis (estático y dinámico). Con base en los extensos estudios paramétricos, se proponen las siguientes limitantes para el uso del método simplificado de análisis en función de la excentricidad estática calculada en planta $\left(e_{s}\right)$ conforme se recomienda hacer en el mismo método simplificado:

- Si se emplea la propuesta de $F_{A E}$ para comportamiento totalmente elástico, la excentricidad estática máxima que debe permitirse es del $5 \%$ o menor inclusive.

- Si se emplean los $F_{A E}$ de la NTCM-2004 para comportamiento parcialmente agrietado, asociado al desempeño de diseño esperado en mampostería confinada, la excentricidad estática máxima que debe permitirse es del $5 \%$.

- Si se emplean las propuestas de $F_{A E P A}$ o $F_{A E T A}$ para comportamiento parcialmente agrietado o totalmente agrietado respectivamente, la excentricidad estática máxima que debe permitirse es del $10 \%$.

El estudio paramétrico descrito ha permitido revisar y proponer, con bases sólidas, valores límites de $e_{s}$ razonables para el empleo del método simplificado para cada nivel de desempeño estructural considerado, siendo ésta su aportación más valiosa.

Se puede concluir que las investigaciones hechas y descritas en este trabajo han permitido actualizar al método simplificado de análisis de los reglamentos de diseño por sismo de México, 
proporcionando con base en extensos estudios paramétricos factores de área efectiva para estructuras de mampostería actualizados para tres distintos niveles de desempeño estructural, así como los respectivos valores límite de la excentricidad estática en planta $\left(e_{s}\right)$ para su aplicación. Estas propuestas ya se incorporan en el método simplificado del capítulo de diseño por sismo del nuevo Manual de Obras Civiles de Comisión Federal de Electricidad (MOC-2008).

Por último, cabe señalar que el método simplificado de análisis de los reglamentos mexicanos permite que los muros de carga sean, además de mampostería, para los cuales ha sido realmente estudiado, también de concreto reforzado, placas de acero, compuestos de estos dos últimos materiales o de madera. Es claro que los factores de área efectiva aquí propuestos para la condición de estado límite de colapso no están calibrados para estos otros materiales, por lo que deberán tomarse sólo como valores indicativos. Por otra parte, será necesario realizar más investigaciones específicas para calibrar factores de área efectiva adecuados para este tipo de materiales, para incluirlas en futuras versiones de los reglamentos de diseño sísmico de México.

\section{AGRADECIMIENTOS}

Los autores agradecen a la Dirección de Obras del Gobierno del Distrito Federal por su patrocinio parcial en las fases iniciales de esta investigación. Jesús Cano Licona y Arturo López Blancas agradecen el apoyo otorgado por Conacyt que les permitió involucrarse en este proyecto de investigación en el desarrollo de sus tesis de maestría.

\section{REFERENCIAS}

Arias, J G, A Vázquez y S M Alcocer (2004), "Respuesta dinámica de modelos a escala 1:2 de viviendas de mampostería confinada de uno y tres pisos, ensayados en mesa vibradora", Memorias, XIV Congreso Nacional de Ingeniería Estructural, Acapulco, CDROM, Artículo II-05, pp. 1-6, noviembre.

Bazán, E y R Meli (1998), Diseño sísmico de edificios, primera edición, editorial Limusa.

Cano, J (2005), "Propuesta de modificación al método simplificado de análisis sísmico de las Normas Técnicas Complementarias para Diseño y Construcción de Estructuras de Mampostería", Tesis de Maestría, División de Estudios de Posgrado de la Facultad de Ingeniería, Universidad Nacional Autónoma de México, marzo.

Cano, J y A Tena (2005), "Diseño sísmico de estructuras de mampostería para distintos niveles de desempeño estructural con base en adecuaciones propuestas al método simplificado de análisis", Memorias, XV Congreso Nacional de Ingeniería Sísmica, México, DF, CDROM, Artículo No. X01, pp. 1-20, septiembre.

Cortés, J A (2009), "Evaluación de la flexibilidad de diafragma para sistemas de piso utilizados en estructuras de mampostería", Proyecto Terminal II, Departamento de Materiales, Universidad Autónoma Metropolitana, agosto.

Damy, J. E. (1986), Apuntes del curso: Tópicos estructurales, División de Estudios de Posgrado de la Facultad de Ingeniería, UNAM.

Damy, J. E. y S. M. Alcocer (1987), "Obtención del centro de torsión de edificios", Memorias, VI Congreso Nacional de Ingeniería Estructural, Querétaro, México, pp. C-60 a C-67. 
Revisión y actualización del método simplificado de análisis de estructuras de mampostería de los reglamentos de ...

Flores, L y S M Alcocer (2001), "Estudio analítico de estructuras de mampostería confinada", Informe Técnico, Coordinación de Investigación, Área de Ingeniería Estructural y Geotecnia, Cenapred, ISBN: 970-628-606-3.

López, A (2007), "Estudio de las limitantes por torsión impuestas al método simplificado de análisis de estructuras de mampostería", Tesis de Maestría, División de Ciencias Básicas e Ingeniería, Universidad Autónoma Metropolitana Azcapotzalco, marzo.

NTCS-77 (1977), "Normas Técnicas Complementarias para Diseño por Sismo", Gaceta Oficial del Distrito Federal, marzo.

NTCS-2004 (2004) "Normas Técnicas Complementarias para Diseño por Sismo", Gaceta Oficial del Distrito Federal, Tomo II, No. 103-BIS, octubre, pp. 55-77.

NTCM-77 (1977), "Normas Técnicas Complementarias para Diseño y Construcción de Estructuras de Mampostería", Gaceta Oficial del Distrito Federal, marzo.

NTCM-2004 (2004), "Normas Técnicas Complementarias para Diseño y Construcción de Estructuras de Mampostería", Gaceta Oficial del Distrito Federal, octubre.

MOC-93 (993), Manual de diseño de obras civiles. Diseño por sismo, Instituto de Investigaciones Eléctricas, Comisión Federal de Electricidad, México.

MOC-2008 (2009), Manual de diseño de obras civiles. Diseño de estructuras de edificios, Instituto de Investigaciones Eléctricas, Comisión Federal de Electricidad, México, diciembre.

Ruiz, J (1995), "Reparación y refuerzo de una estructura tridimensional de mampostería confinada de dos niveles a escala natural", Tesis de Maestría, División de Estudios de Posgrado de la Facultad de Ingeniería, Universidad Nacional Autónoma de México, diciembre.

Ruiz, J y S M Alcocer (1998), "Desempeño experimental de estructuras de mampostería confinada rehabilitadas mediante el uso de malla de alambre", Revista de Ingeniería Sísmica, SMIS, No. 59, pp 59-79, julio-diciembre.

Taranath, B S (1988), Structural analysis and design of tall buildings, primera edición, McGraw Hill, Nueva York, ISBN 0-07-062878-5.

Tena, A y M A Pérez (2000), "Importancia de las deformaciones por cortante en la ubicación de los centros de torsión de entrepiso en edificios estructurados con base en muros", Memorias, XII Congreso Nacional de Ingeniería Estructural, León, CDROM, noviembre.

Tena, A, M A Pérez y J Cano (2002), "Propuesta de modificación a la estimación de las fuerzas cortantes del método simplificado de análisis", Memorias, XIII Congreso Nacional de Ingeniería Estructural, Puebla, CDROM, Artículo No. 156, pp. 1057-1068, noviembre.

Tena, A (2007), Análisis de estructuras con métodos matriciales, primera edición, editorial Limusa, ISBN-13:978-968-18-6980-9, septiembre.

Tena, A y A López (2006), "Revisión de la excentricidad límite del método simplificado de análisis de estructuras de mampostería del RCDF vigente", Memorias, XV Congreso Nacional de Ingeniería Estructural, Puerto Vallarta, Jalisco, CDROM, pp. 1-22, noviembre.

Tena-Colunga, A y M A Pérez-Osornio (2005), "Assessment of shear deformations on the seismic response of asymmetric shear wall buildings", ASCE Journal of Structural Engineering, Vol. 131, No. 11, pp. 1774-1779, noviembre.

Tena-Colunga, A, A Juárez-Ángeles y V M Salinas-Vallejo, (2009), "Cyclic behavior of combined and confined masonry walls", Engineering Structures, Vol. 31, No. 1, pp. 240-259. 
Tena, A y J A Cortés (2009), "Evaluación de la condición de diafragma rígido o flexible para el empleo del método simplificado en estructuras de mampostería", Memorias, XVII Congreso Nacional de Ingeniería Sísmica, Puebla, Puebla, CDROM, noviembre. 